\title{
Amplitude and Angular Scintillations of the Radio Source Cygnus-A Observed at Boulder, Colorado ${ }^{1}$
}

\author{
R. S. Lawrence, J. L. Jespersen, and R. C. Lamb
}

\begin{abstract}
(November 3, 1960)
Variations in the apparent flux and position of the radio source Cygnus-A were recorded at 53 and 108 megacycles per second using a two-element, phase-sweeping interferometer located at Boulder, Colo. An ionospheric sounder operating at Ellsworth, Nebraska, provided, for a few hours each day, simultaneous vertical-incidence measurements on the ionosphere at its intersection with the line of sight from Boulder to the radio star. Amplitude scintillations observed at Boulder over a twelve-month period are compared with ionograms taken at Ellsworth. Positive correlation is found between amplitude scintillations and spread $F$, while no significant correlation is found with sporadic $E$.

Detailed analysis of the scintillations indicates that the probability distribution of the amplitude can be represented by the Rice probability distribution function. The zenithangle dependence of the amplitude scintillations does not agree with a theory based upon isotropic ionospheric inhomogeneities.

The root-mean-square value of angular scintillations is proportional to the square of the wavelength, in accord with a theory of diffraction by ionospheric irregularities. Comparison of angular scintillations with amplitude scintillations indicates that, for elevation angles of $15^{\circ}$ to $50^{\circ}$, the region of the ionosphere responsible for scintillation lies near the border between the Fresnel and Fraunhofer diffraction regions for both frequencies.

Slow, irregular angular variations are commonly observed in the daytime at both frequencies. These variations are attributed to lens-like ionospheric irregularities having dimensions as large as 200 kilometers.
\end{abstract}

\section{Introduction}

It has been recognized since 1950 that the apparent fluctuations in intensity of discrete radio sources arise in the ionosphere. In a manner analogous to the twinkling and dancing of visible stars, these "amplitude scintillations" are accompanied by "angular scintillations", which are fluctuations in the apparent position of the radio source.

Radio-star scintillations offer a convenient means for studying the irregular structure of the ionosphere. The $F$ region is generally believed to play an important role in the production of scintillations, but our knowledge of the effect of the E regio $\mathrm{I}$ is confused by incomplete and apparently conflicting evidence. One of the purposes of the experiment reported here was to improve our understanding of the ionospheric conditions and levels responsible for scintillations. The experiment was designed to be particularly sensitive to any relationship between scintillations and the $E$ region. The results have indicated little or no such relationship, but do show a connection between scintillations and spread echoes in the $F$ region. In addition to the comparison of scintillations and ionospheric phenomena, detailed analysis has been made of the scintillations themselves. In connection with this, some statistical aspects of amplitude scintillations are found to disagree with a theory based upon plane-earth geometry and spher-

\footnotetext{
1 Contribution from Central Radio Propagation Laboratory, National Bureau of Standards, Boulder, Colo.
}

ical irregularities whereas the statistical variations in angular scintillations agree with this theory.

A third aspect of the experiment has been the observation and study of slow angular variations in the apparent position of the source. These variations are due to refraction by individual large irregularities, in contrast to amplitude and angular scintillations which are due to the diffraction effects of a number of smaller irregularities.

A description of the planning and instrumentation for the experiment, along with a brief summary of the pertinent literature, has already appeared [Lawrence, 1958]. In addition, two review papers [Booker, 1958; Little et al., 1956] are available which outline present knowledge concerning scintillations and which describe the usefulness of scintillation observations as a tool for studying the irregular structure of the ionosphere.

\section{Description of the Observations}

The radio source Cygnus-A has been observed at Boulder (latitude $40^{\circ} 7^{\prime} .7 \mathrm{~N}$, longitude $105^{\circ} 14^{\prime} .3 \mathrm{~W}$ ) at frequencies of 53 and $108 \mathrm{Mc} / \mathrm{s}$, using a two-element, phase-sweeping interferometer. The phasesweeping system permits simultaneous observation of the apparent flux from the radio source and of the relative phase of the radio waves at the two antennas. Amplitude scintillations are simply variations in the flux, while angular scintillations may be deduced from the known spacing of the antennas, the general direction of the radio source, and the variations in relative phase of the radio waves. 
From February 1958 through February 1959 observations were obtained regularly during the three or four hours each day following the rising of Cygnus-A. Figure 1 shows how the time of such observations varies throughout the year and indicates the seasons during which the effective ionosphere was sunlit.

The two antennas are separated by $475 \mathrm{~m}$ on a horizontal, east-west baseline and the resulting interferometer lobes are of nearly constant spacing over the region of the sky in which the observations were made. At a radiofrequency of $108 \mathrm{Mc} / \mathrm{s}$ the lobe spacing is about $0.4 \mathrm{deg}$; at $53 \mathrm{Mc} / \mathrm{s}$ it is twice as great. The measured component of angular scintillations is, of course, perpendicular to the lobes. This means that, near the horizon, angular scintillations can be observed only in the horizontal direction, while at an elevation angle of about $35^{\circ}$ the horizontal and vertical components of angular scintillations are observed with equal sensitivity.

At the time when Cygnus- $\mathrm{A}$ is about $15^{\circ}$ above the north-east horizon at Boulder, the line of sight intersects the $E$ region over Ellsworth, Nebraska. During the appropriate portion of each day, an ionospheric sounder was operated at Ellsworth at 5-min intervals. As the radio source rises, the intersection point of the line of sight with the $E$ region moves toward Boulder, and the intersection with the $F$ region passes within about $100 \mathrm{~km}$ of Ellsworth. Figure 2 is a map of the Boulder-Ellsworth area showing the plan view of the intersection point for various ionospheric heights. The numbers along the curves indicate the local sidereal time (LS'T) at Boulder.

\section{Instrumentation}

The instrumentation for the scintillation measurements has already been described in some detail [Lawrence, 1958], but is briefly reviewed here. The two frequencies, 53 and $108 \mathrm{Mc} / \mathrm{s}$, are observed with the same antennas and with similar receiving and recording systems.

Antennas. The two 40-ft paraboloidial reflecting antennas are modifications of standard 28 -ft paraboloids manufactured by the D. S. Kennedy Company. The 6 -ft radial extensions were designed and constructed at NBS. The antennas, separated by 475 $\mathrm{m}$, are equatorially mounted and equipped with sidereal drives for tracking radio stars. The dipole feed, with four reflecting elements, is designed for use simultaneously at both frequencies. A multicoupler permits the use of a single coaxial transmission line from each antenna to the central receiving shelter. No preamplifiers are used at the antennas.

Receivers. At each frequency, the dual receiving channels are connected together as a Ryle interferometer [Ryle, 1952]. A rotary phase shifter has been added to one channel so that the interferometer pattern may be swept across the sky. This phase shifter, usually driven at a uniform rate of $10 \mathrm{rpm}$, converts the system to a "phase-sweeping" interferometer. A "phase-tracking" feature was included in the original design, whereby the interferometer pattern was servo-controlled to follow the apparent motion of the radio source. This feature permits the direct recording of angular scintillations.

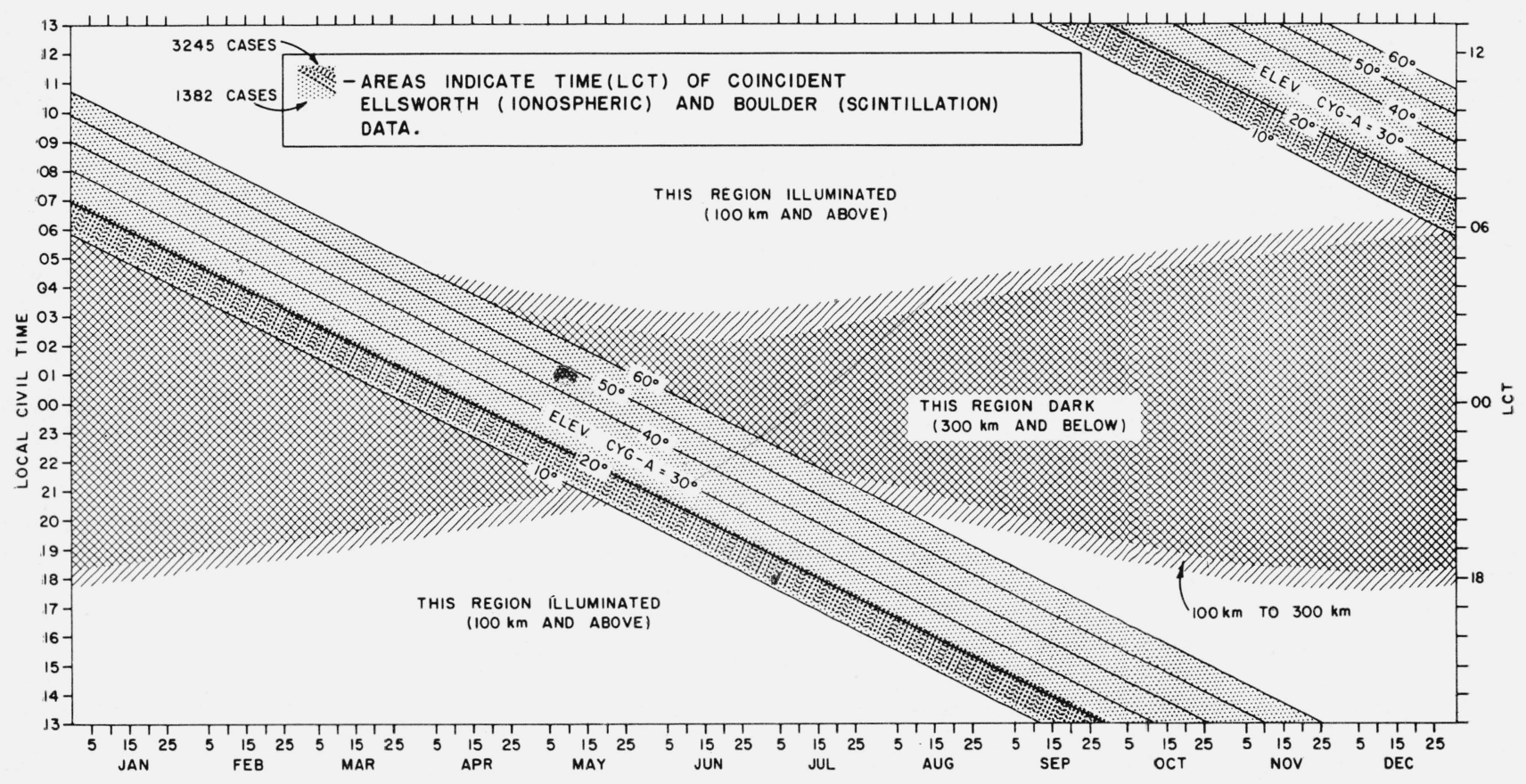

Figure 1. Time of observation of Cygnus-A, and state of illumination of the ionosphere. 


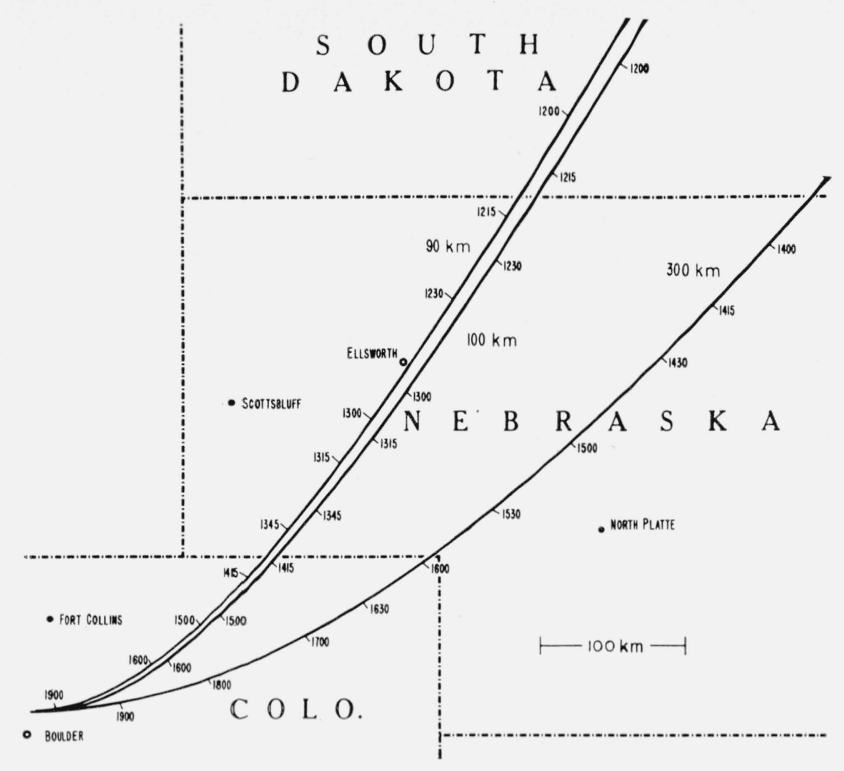

Figure 2. The variation, with local sidereal time at Boulder, of the position at which the line-of-sight from Boulder to Cygnus-A penetrates the ionosphere.

The three curves apply to ionospheric heights of 90,100 , and $300 \mathrm{~km}$.

However, it keeps a zero-crossing of the interferometer pattern always on the source and so prevents recording of amplitude information. As a result, it is difficult to distinguish authentic angular scintillations from the random behavior of the servo loop which accompanies deep amplitude fades. Accordingly, all of the observations reported here were made in the phase-sweeping mode.

The receivers have bandwidths of $500 \mathrm{kc} / \mathrm{s}$, and were operated with output time constants of one second. The double-conversion superheterodyne system uses intermediate frequencies of 31.5 and $7.5 \mathrm{Mc} / \mathrm{s}$.

Digital recorder. In order to permit the use of a computer for processing the data, chart recordings were supplemented by simultaneous digital recordings on punched paper tape. A simple, meter-type analog-to-digital converter sampled the output from each interferometer once per second, and punched the two outputs alternately on a single paper tape. The six-bit binary code provides a full-scale accuracy of 1.5 percent, approximately the same as careful manual scaling of a pen recording.

\section{Data Analysis}

The following is a summary of data-analysis procedures. The appendices and the following paper by the same authors, contain a detailed explanation of the procedures outlined in this section.

The observations made in the progress of this experiment consist of scintillation records from the phase-switch, phase-sweep interferometer located at Boulder, and of ionograms from an ionospheric sounder located at Ellsworth, Nebr.

Ionograms. Vertical-incidence, sweep-frequency ionospheric records were taken at $5 \mathrm{~min}$ intervals for $1 \mathrm{hr}, 1230$ through $1330 \mathrm{LST}$ each day during the time that the ray path from Cygnus-A passed through the $E$ region $(100 \mathrm{~km})$ near Ellsworth. After this period, ionograms were made each halfhour for the next $3 \mathrm{hr}$. These ionograms have been scaled for critical frequencies, minimum virtual heights, and for the occurrence of special features, particularly for the absence or presence of spread $F$ and of the various types of sporadic $E$. Each type of information from the ionogram was converted to an appropriate single-digit scale and punched in a separate column of a uniquely identified IBM card. Simultaneous scintillation information, described below, was later added to each card.

Scintillation records. The scintillation records, both analog and digital, consist of a sine wave ("carrier") having a frequency determined by the rate of lobe sweeping and an amplitude and phase which are modulated by the ionosphere. For the present experiment several methods were used to extract, from the digital records, the amplitude and phase modulation. However, all of these methods depend upon the fact that the envelope of the carrier is affected only by ionospheric amplitude effects and that the times of zero-crossings are affected only by ionospheric phase effects. Intermediate points on the carrier are affected by both. The power index

$$
\left(\frac{\Delta P}{P}\right) \equiv \overline{\left|\frac{P-\bar{P}}{\bar{P}}\right|}
$$

and the amplitude index

$$
\left(\frac{\Delta A}{A}\right)^{2} \equiv \overline{\left(\frac{A-\bar{A}}{\bar{A}}\right)^{2}}
$$

have been computed to measure amplitude effects. $P$ is the power from the discrete source and may be read directly from the envelope of the carrier since a square-law detector was used in the scintillation receivers. $A=\sqrt{P}$, and a bar denotes an average over the record. The reason for computing both of these quantities will be shown in a later section.

The variance of the angular scintillations, $\overline{\theta^{2}}$, was computed as a measure of phase effects. For any given elevation angle, $\overline{\theta^{2}}$ can be deduced from the observed value of $\sigma_{\alpha}$, the standard deviation of the phase difference, $\alpha$, between the two ray paths to the interferometer. Variations in $\alpha$ may be determined directly from the variations in the interval of time between successive zero-crossings. (See following paper by the same authors.)

The first attempt to measure amplitude scintillations was to assign visually one of five possible categories to each 5-min section of the scintillation records (see appendix 1). Those sections for which there were no visible variations in the envelope of the carrier were assigned a "depth index" of 1 , those for which there were shallow variations in the envelope were assigned a depth index of 2 , etc., to the depth index 5 for records with amplitude effects so 
deep that the lobes disappeared. Several examples of each of these categories of records were scaled by hand so that an estimate could be made of the range in values of $(\Delta A / A)^{2}$ and of $(\Delta P / P)$ associated with each category (see the table in appendix 1 ).

A second approach was to recover $P$ and $\alpha$ from the carrier and then to plot these two quantities as functions of time. The resulting graphs are referred to as power plots and phase plots respectively. Two different methods were used ("zero-crossing", and "filter") to make this recovery (see following paper). The power and phase plots may be used to compute the quantities $(\Delta P / P),(\Delta A / A)^{2}$, and $\overline{\theta^{2}}$, and it is also possible to use them to determine the power spectra of amplitude and angular scintillations.

Some of the records, because of large amplitude and/or phase effects, are not amenable to analysis by either the "zero-crossing" or "filter" methods. These records were analyzed using a statistical approach which does not give detailed power and phase plots but which gives the quantities $P_{n}$ and $\sigma_{d}$, quantities which may be related to $(\Delta A / A)^{2}$ and $\overline{\theta^{2}}$ (see following paper by the same authors).

\section{Results and Discussion}

The relation between amplitude scintillations and ionospheric parameters. Figure 3 displays the distribution with elevation angle of the Ellsworth ionograms and the coincident scintillation records which were used to investigate the correlation between the two types of observations. Figure 4 shows a definite correlation at both radiofrequencies between the depth of amplitude scintillation and the occurrence of spread $F$. Here the Fisher $Z_{r}$ function [Walker and Lev, 1953] is plotted at the weighted mean elevation angles of the 10-deg class intervals shown in figure 3. The upper scale gives, for each elevation angle, the ionospheric height at which the line of sight to Cygnus-A comes closest to the zenith at

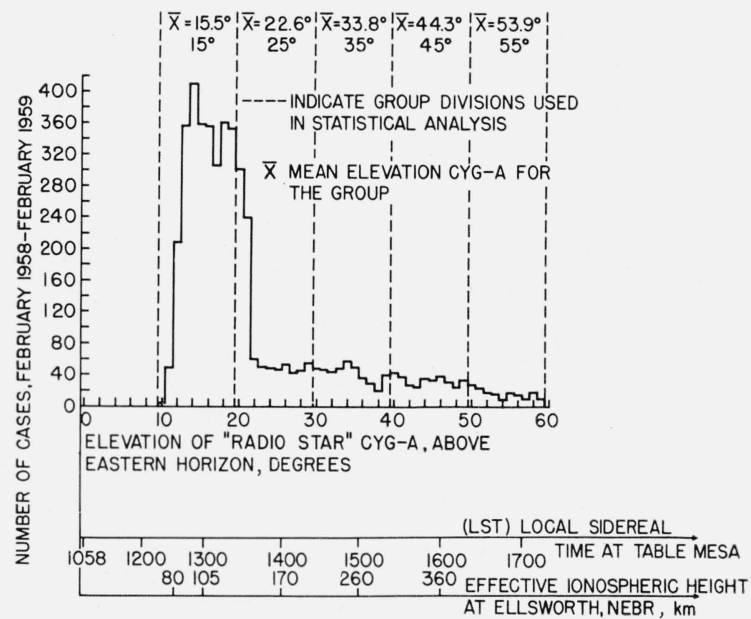

FIGURE 3. The grouping for statistical analysis, with elevation angle and sidereal time, of the scintillation data.

The "effective ionospheric height" is the height at which the line-of-sight from Boulder to Cygnus-A comes closest to the zenith at Ellsworth, Nebr.
Ellsworth. $N$ is the number of samples used in the computation of each value of $Z_{r}$, and the vertical bar through each point has length $2 \sigma_{z_{r}}$, twice the expected standard deviation of the correlation coefficient. Each graph is derived using the total data sample. Division of the data into sunlit and dark ionospheric groups results in correlations (not shown) which have, in general, a value of $Z_{r}$ which is 0.1 higher (dark ionosphere) and 0.1 lower (sunlit ionosphere) than the correlations shown, with correspondingly larger values of $\sigma_{z_{r}}$.

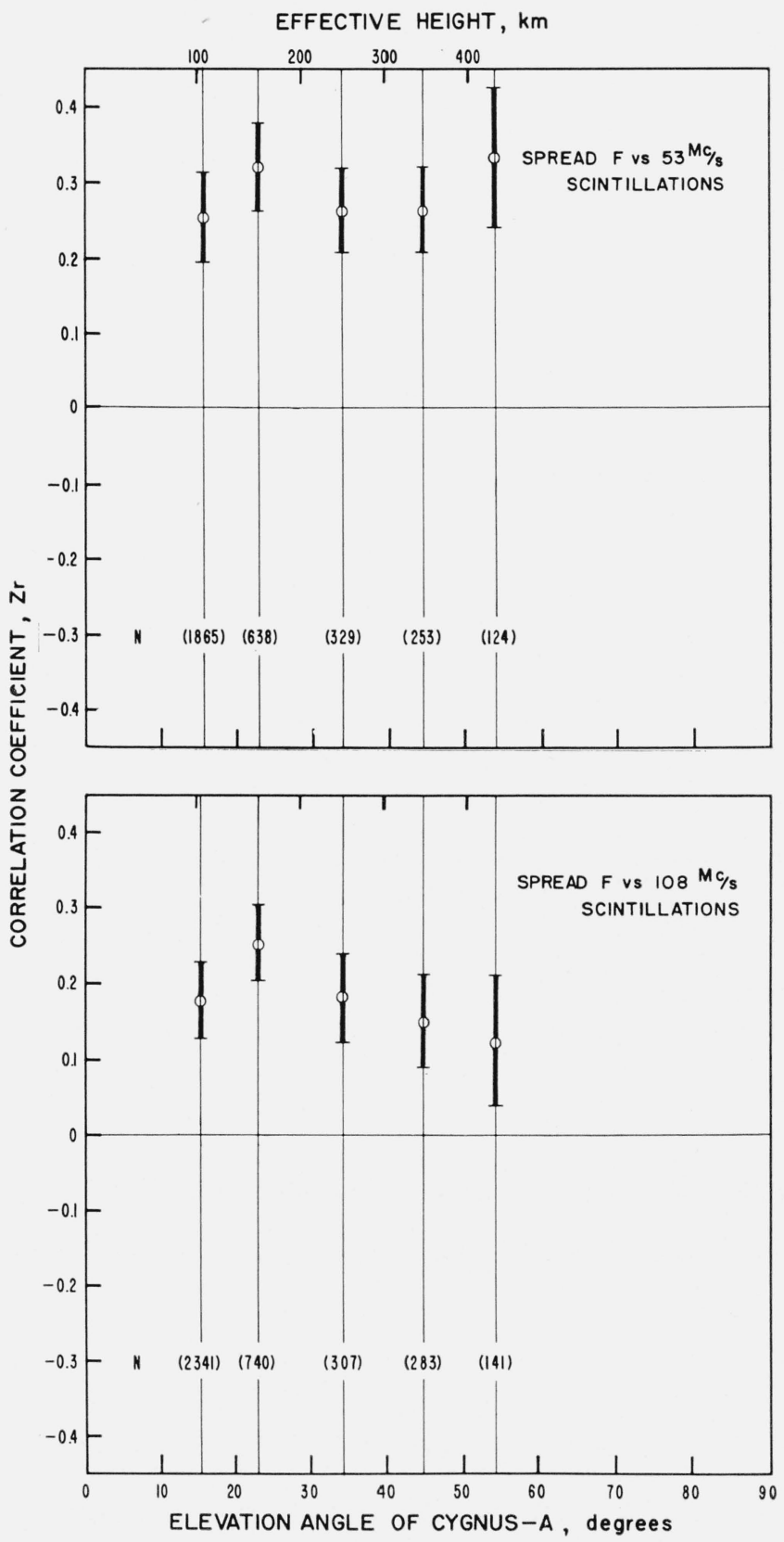

FIgURE 4. The correlation between amplitude scintillations and spread F, as a function of source elevation for each radio frequency.

$\mathrm{N}$ is the number of cases. 
In the same manner, figure 5 displays the correlation, using the total data sample, between the depth of amplitude scintillations and the occurrence of sporadic $E$. Division of the data according to sunlit and dark ionosphere, as well as by season, resulted in no significant change from the results shown.

Other ionospheric parameters showed little or no correlation with the depth of amplitude scintillations. In particular, there was no significant correlation, at either radiofrequency, with $f_{0} F 2$, foEs, $h^{\prime} E s$, or $f_{\min }$. Correlation with $h^{\prime} F$, the minimum virtual height of the $F$ region, resulted in a $Z_{r}$ of

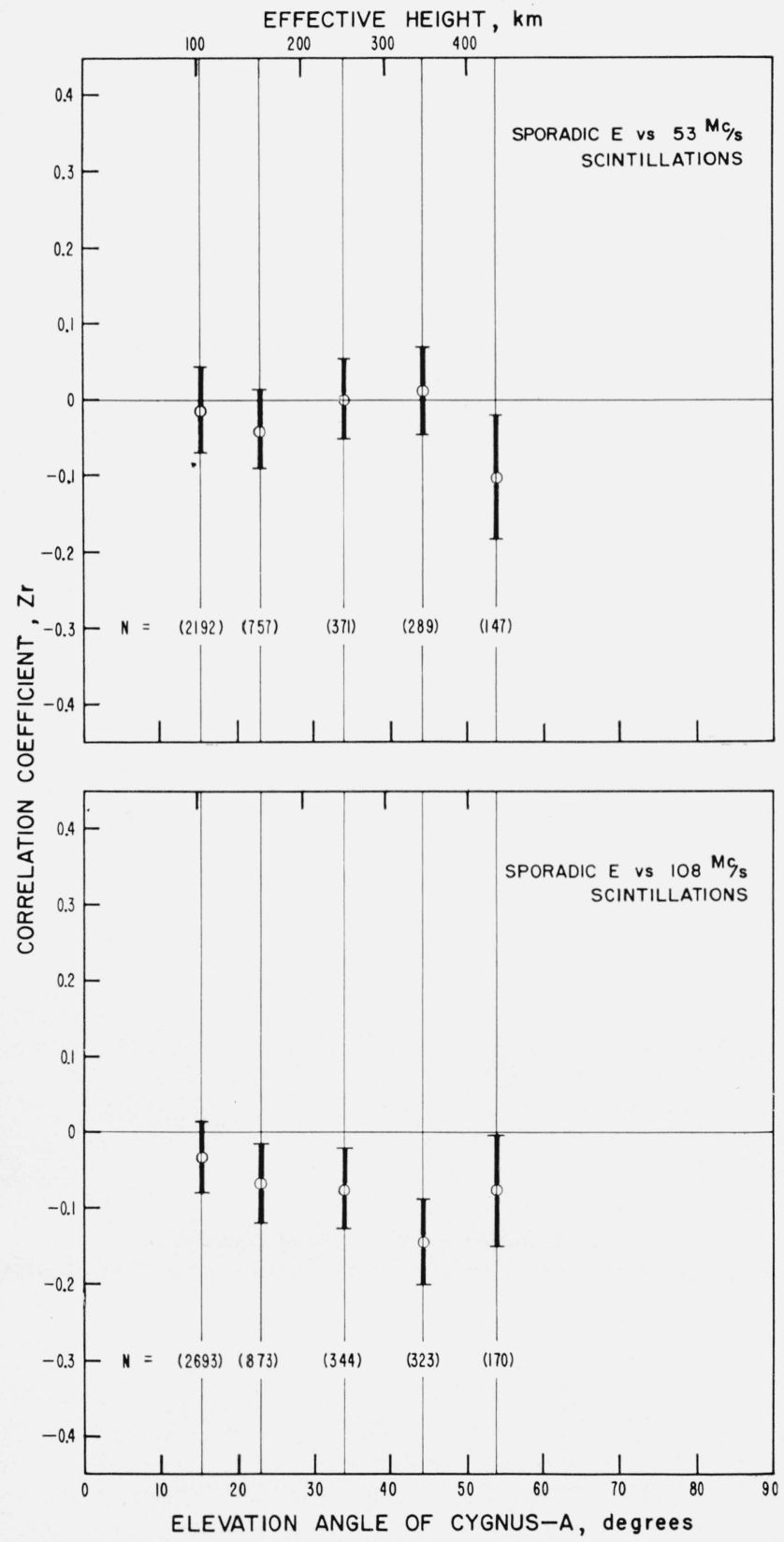

Figure 5. The correlation between amplitude scintillations and sporadic E, as a function of source elevation for each radio frequency. about 0.2 to 0.3 at $53 \mathrm{Mc} / \mathrm{s}$ and a $Z_{r}$ of about 0.1 to 0.2 at $108 \mathrm{Mc} / \mathrm{s}$, at $F$-region effective heights.

Many, but not all, previous observers have found scintillations to be correlated with the phenomenon of spread- $F$ ionospheric echoes. In the early days of scintillation study, Ryle and Hewish [1950] and Little and Maxwell [1951] both found such relationships, while Mills and Thomas [1951] found positive correlation with an " $F$-region index". Later Bolton, Slee, and Stanley [1953] reported positive correlation during the winter months; as did Wild and Roberts [1956a] for nighttime scintillations, although in both cases the ionosonde was located many hundreds of kilometers from the point of $F$-region penetration. Both Dueño [1955] and Hartz [1958] failed to find correlation with ionosonde data taken at points appreciably far from the optimum for $F$ region penetration, although $\mathrm{Hartz}$ and, more recently, Dagg [1957] noted that the diurnal variations are similar. Briggs [1958], on the other hand, has recently studied the correlation between scintillations and spread $F$ to deduce the spatial extent and the height of the region responsible for scintillations. Wright, Koster, and Skinner [1956]; and Koster, [1958] have noticed a quite high positive correlation with equatorial spread $F$, but this may be a different phenomenon from the spread $F$ at temperate latitudes.

The diurnal variations of spread $F$ and scintillations are generally reported to be similar, and a comparison between figures 6 and 7 indicates that this is clearly the case for the present experiment. This fact alone might explain some of the correlations which have been reported. In other cases, a disadvantageous location of the ionosonde may have reduced or destroyed the correlation. We have attempted to avoid both pitfalls, first, by removing the average diurnal and seasonal variation from both the scintillation data and the spread $F$ observations (see appendix 2), and second, by locating the ionosonde at Ellsworth, Nebr. Although Ellsworth was chosen specifically for E-region observations, the location should provide quite reliable $F$-region data for elevation angles of Cygnus-A in the range from 25 to 65 deg above the northeastern horizon at Boulder.

The positive correlation between spread $F$ and the depth of amplitude scintillations, as shown by figure 4, agrees with many of the results described previously. The location of the ionosonde, although not ideal for $F$-region effects, is quite satisfactory if, as has been reported [Briggs, 1958; Koster, 1958; Yeh and Swenson, 1959; and Reid, 1957], the irregularities responsible for spread $F$ occur in patches 300 to $500 \mathrm{~km}$ in lateral extent. The data revision process, which removed the diurnal and seasonal variation from the ionospheric and scintillation parameters, insures that any correlation which remains must be the result of detailed hour-to-hour or dayto-day correlation between the two variables (see appendix 2 ).

The correlation, for a given elevation angle of Cygnus-A was generally higher at $53 \mathrm{Mc} / \mathrm{s}$ than at $108 \mathrm{Mc} / \mathrm{s}$. This result might have been anticipated 

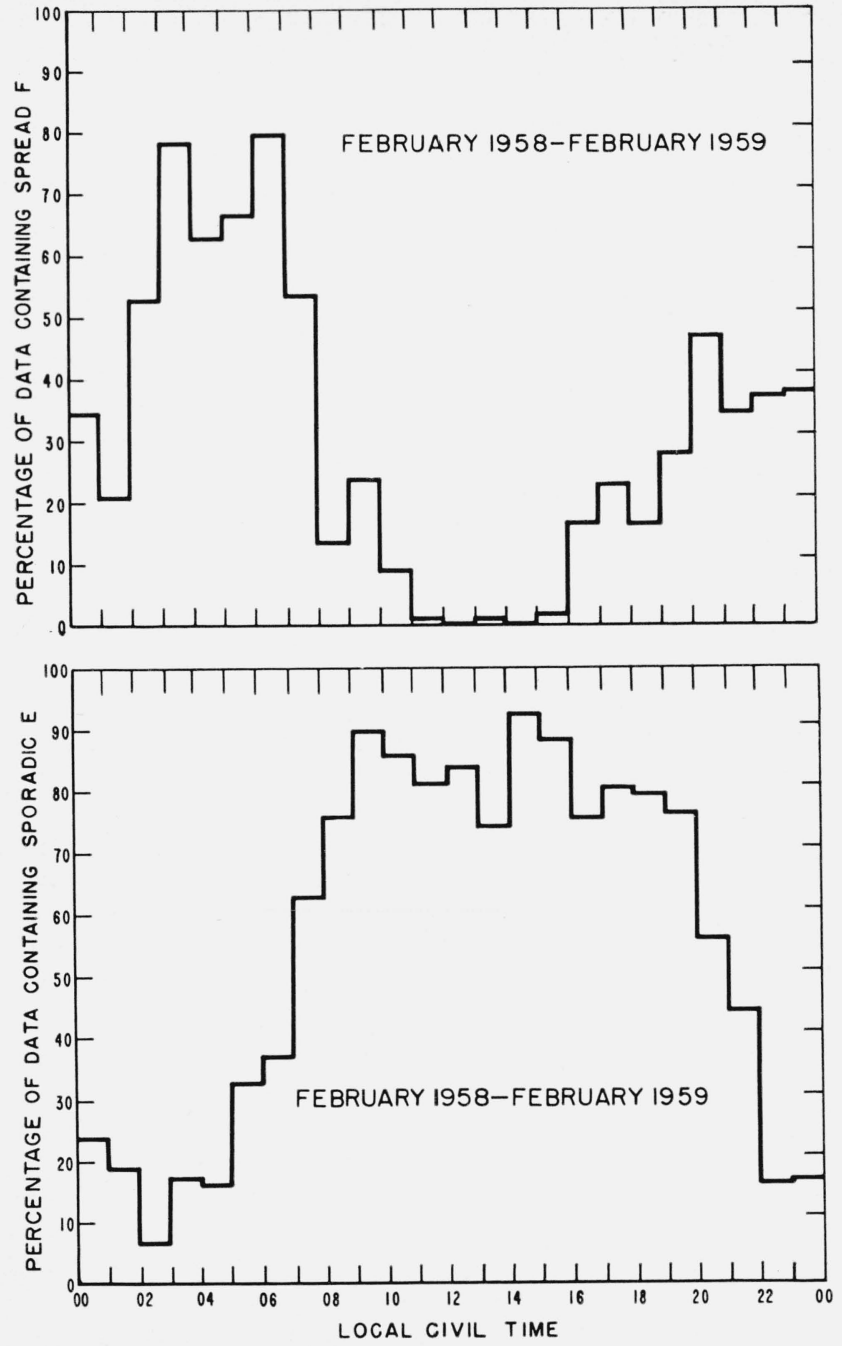

FIGURE 6. The frequency of occurrence, averaged over a year, of spread $\mathrm{F}$ and of sporadic $\mathrm{E}$ at the times of scintillation observations.

since the refractivity of the ionosphere varies inversely with the square of the radio-wave frequency. The nearly constant value of correlation, at either radiofrequency, over the whole range of elevation angles, indicates that the patches of spread $F$ cover large areas. This result has the unfortunate effect of making height determination, by the method used in this experiment, impractical. The correlation was considerably higher at nighttime than in the daytime, and this result tends to reinforce the idea that scintillations are related to spread $F$, since spread $F$ reaches a maximum shortly after local midnight. It is possible that this variation of correlation with local time is partly a result of our inability to remove completely the effect of diurnal and seasonal trends. It is clear that correlations of this type could be found simply by comparing trends, even though there is little, or no, detailed correlation present.

The correlation between scintillations and spread $F$ is significant, but still far from perfect; however, two features of our experimental technique have

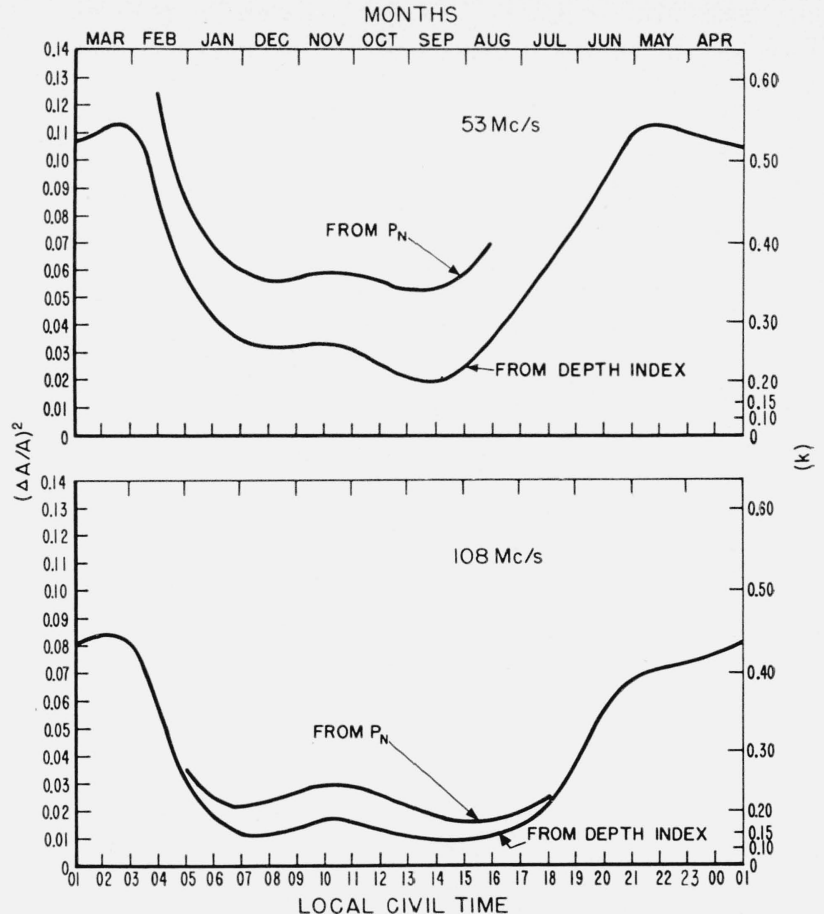

Figure 7. Hourly average values of scintillation depth index and of the statistical quantity $P_{n}$, both converted to $(\Delta A / A)^{2}$ and to the "noise-to-signal ratio", $\mathrm{k}$.

tended to reduce the apparent correlation. First, Ellsworth is not ideally located for $F$-region comparisons; second, and perhaps more important, we have used a spread- $F$ index which depends only upon the absence or presence of spread $F$, not upon its intensity. By themselves, these two effects would be insufficient to reduce an otherwise perfect correlation to the observed value of about 0.3 . Therefore, it seems probable that scintillations sometimes arise in ionospheric regions, such as $E$ or upper $F$, which do not produce observable spread- $F$ echoes.

A number of scintillation observers have searched for correlation with E-region phenomena. Mills and Thomas [1951] found no correlation with sporadic E, whereas Dueño [1955] and Bolton, Slee, and Stanley [1953] reported a general, not detailed, positive correlation between the occurrence of sporadic $E$ and the occurrence of scintillations. In each case, the ionosonde was located more than 200 $\mathrm{km}$ from the E-region penetration point. Wild and Roberts [1956a] found positive correlation with daytime $f E s$, but no correlation at night. Hartz [1958] working in Canada, found a general negative correlation with sporadic $E$.

Because sporadic $E$ seems to occur in smaller patches than does spread $F$, it is significant that the Ellsworth station was located at precisely the correct place to study correlations at E-region heights. The ray path from Cygnus-A to Boulder passes very near the zenith at Ellsworth at the $100 \mathrm{~km}$ level and is within $100 \mathrm{~km}$ of the zenith for elevation angles in the range from 10 to 22 deg above the northeastern horizon at Boulder. As mentioned previously, 
the data revision process retains any correlation between detailed events but markedly reduces the effect of diurnal and seasonal trends. The results of other workers [Hartz, 1958; Dyce, 1955] as well as those shown in figures 6 and 7 indicate that the diurnal variation of the occurrence of sporadic $E$ and the diurnal variation of the occurrence of scintillations are inversely correlated.

Interim results [Lawrence and Jespersen, 1959] of the present experiment indicated a negative correlation (at E-region heights) between scintillations and sporadic $E$. Those early results were obtained from only the first six months (Feb. 58-July 58) data, and the diurnal and seasonal trends had not been removed. It appears in retrospect that these correlations were produced by the inverse diurnal trends in the two variables. They, as well as the present results, are derived from data taken at 5-min intervals in the relevant range of elevation angles. Accordingly, the sensitivity of the result to any real relationship should be high.

The present results, shown in figure 5, indicate no significant correlation, at either radiofrequency, with sporadic $E$. Although this contradicts the weak negative correlation mentioned in the interim report, we infer that there is no observable, detailed connection between sporadic $E$ and amplitude scintillations at this latitude.

Excepting spread $F$, the only ionospheric parameter to exhibit significant correlation with scintillations was $h^{\prime} F$, the minimum virtual height of the $F$ region. This positive correlation was higher at $53 \mathrm{Mc} / \mathrm{s}$ than at $108 \mathrm{Mc} / \mathrm{s}$ and tended, even more than did the correlation with spread $F$, to be restricted to a range of elevation angles which indicated $F$-region heights. This suggests that the properties of the electron-density profile which determine $h^{\prime} F$ are related to scintillations, but do not have good serial correlation over such large distances as does spread $F$. In addition to this, the correlation may be related to the diurnal and seasonal variation of radio star scintillations, a variation which has been the object of much study [Bolton, Slee, and Stanley, 1953; Hartz, 1958; Dagg, 1957; Briggs, 1958; Koster, 1958; Yeh and Swenson, 1959]. Figure 7 shows the observed variation in $(\Delta A / A)^{2}$. This variation contains a mixture of both seasonal and diurnal effects since only one source, Cygnus-A, was used. Theory indicates [Booker, 1958] that, in the Fresnel (near zone) region, $(\Delta A / A)^{2}$ is proportional to the square of the distance, $z$, between the observer and the diffracting screen (see eq (2)). If $h^{\prime} F$ is related to $z$, then one would expect a correlation between amplitude scintillations and $h^{\prime} F$; also, any seasonal or diurnal variation in $h^{\prime} F$ would produce a correponding variation in $(\Delta A / A)^{2}$. However, $h_{\max } F$ is probably a better measure of $z$ than is $h^{\prime} F$. In view of this possibility, the observed diurnal and seasonal variation in $h_{\max } F$, at this latitude for the period corresponding to the times that scintillation data were taken, was used to obtain $z$ for eq (2), and the resulting variation in $(\Delta A / A)^{2}$ was compared with the observed variation (fig. 7). At 53 $\mathrm{Mc} / \mathrm{s}$ (using the $P_{n}$ curve) the agreement was quite good. No comparison was made at $108 \mathrm{Mc} / \mathrm{s}$ since the available portion of the $P_{n}$ curve shows little variation. The agreement at $53 \mathrm{Mc} / \mathrm{s}$ shows that a hypothetical diffracting screen which always remains at the height of maximum electron density, and whose statistical properties never change, will produce the same diurnal variation in amplitude scintillations as is observed at Boulder. However, such a model seems quite unreasonable since it implies that all the irregularities are forced to move with $h_{\max } F$, and that their intensity, $\Delta N$, is independent of electron density, $N$.

Amplitude scintillations. The power plots obtained from the "zero-crossing" and "filter" methods provide a convenient basis for detailed analysis of amplitude scintillations. Using the method described by Tukey [1949], power spectra extending over the frequency range 0.005 to $0.17 \mathrm{c} / \mathrm{s}$ have been calculated from the power plots. Four such spectra, together with the corresponding values of $(\Delta A / A)^{2}$, are displayed in figure 8 . The ordinate value is the common logarithm of the variance per unit frequency interval, expressed in units of the square of the average power. Since the spectra come from power plots, and these cannot be obtained for the highly disturbed records, the two upper curves in figure 8 , although they are the highest spectra available, should be taken as typical of only moderate scintillation activity. The two lower spectra were derived from data with little scintillation activity, and are close to the noise level.

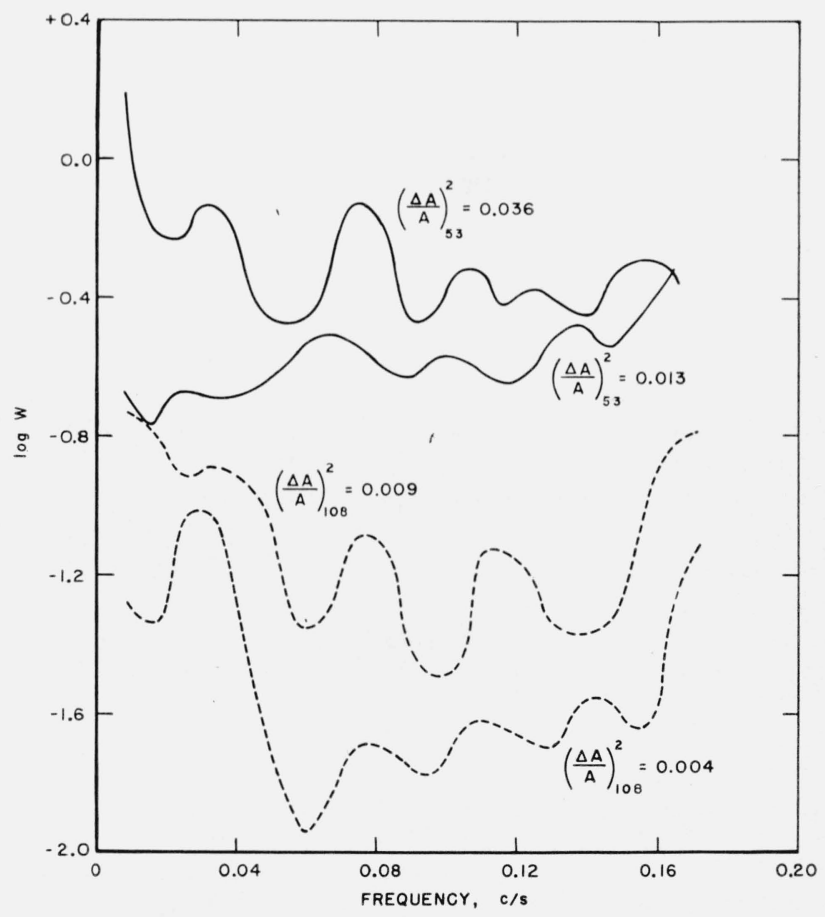

FIGURE 8. The figure shows the highest and lowest observed amplitude power spectra for each radio frequency.

The lower level at each frequency indicates the background "noise" level. The units of $W$ are the variance per unit frequency interval expressed in units of the square of the average power. 
Each pair of spectra in figure 8 was derived from simultaneous power plots at the two radiofrequencies. Comparison of these spectra, and of many others like them, indicates that there is no detailed correlation in the frequency domain. (The quasiperiodic variation superposed on the spectra is caused by the finite length of the data samples and should not be misconstrued as an indication of correlation between the spectra [Blackman and Tukey, 1959].) No correlation is to be expected for scintillations which result from a diffraction process because the distance, measured in wavelengths, between the observer and the diffracting screen is very different for the two radiofrequencies. Refraction, on the other hand, should cause detailed correlation. Thus, we conclude that, at $108 \mathrm{Mc} / \mathrm{s}$, refraction plays no observable role in the production of the amplitude scintillations we observe, in contrast to the observations made at lower frequencies by Wild and Roberts [1956b.]. The lack of correlation also indicates that there are no correlated instrumental errors, such as might be introduced by variations in the power-line voltage or by movements of the antennas.

Excepting the short-period fluctuations which are the effect of finite sample length, the power spectra follow a definite pattern. Typically, log $W$ decreases linearly with frequency, up to a frequency of about $0.1 \mathrm{c} / \mathrm{s}$, and becomes nearly constant thereafter. The negative slope of the decreasing portion of the spectrum is directly related to the "fading period," $t$, which was used in a previous study of the amplitude scintillations [Lawrence and Jespersen, 1959]. (In that study, $t$ was defined as twice the average interval between crossings of the average power level.) Although the upper example in figure 8 does not seem to concur, the negative slopes of pairs of spectra derived from simultaneous scintillation records made at the two radiofrequencies, are generally very similar. Such independence between fading rate and frequency is to be expected for scintillations which arise from a diffraction process.

The power spectra are nearly flat for frequencies greater than $0.1 \mathrm{c} / \mathrm{s}$, but the shape of this portion of the spectrum is severely influenced by the discrete sampling of the original data. When a function, $x(t)$, is sampled at equal intervals of time, $\Delta t$, then the frequencies $2 f_{N} \pm f, 4 f_{N} \pm f, 6 f_{N} \pm f$, etc. are not distinguishable from the frequency $f$ [Blackman and Tukey, 1959]. Here, $f_{N}$, the Nyquist frequency, is defined as $f_{N}=1 / 2 \Delta t$. Thus, if frequencies higher than $f_{N}$ are present in the data they will contribute their energies to lower frequencies in the spectrum. This phenomenon is frequently described as "aliasing" or "folding" of the higher frequencies into the range of interest of the power spectrum.

As mentioned previously, the power spectra could be computed only for records which displayed moderate, slow scintillations. On such records, most of the variance due to scintillations is composed of frequencies less than $f_{N}$, and those frequency components greater than $f_{N}$ will be largely due to white noise. The effect of white noise, when folded back into the spectrum, has been examined and is found to increase the general level of the spectrum by a nearly constant amount. In order to avoid aliasing the power spectra, one would ordinarily use appropriate low-pass filters to remove the higher frequencies before sampling. However, in the case of the "zero-crossing" method (see following paper by the same authors), the necessary filter would have an upper frequency cutoff equal to the phase-sweeping frequency and would thus remove the signal.

The power plots have also been analyzed in terms of $(\Delta A / A)^{2}$ and $(\Delta P / P)$, the amplitude and power indices [Lawrence and Jespersen, 1959]. The relation between $(\Delta A / A)^{2}$ and $(\Delta P / P)$ depends upon the distribution law obeyed by the amplitude, $A$, of the radio wave. Thirty-one records selected at random were used to produce the dots shown in figure 9 , where it may be noted that the observed values of amplitude index $(\Delta A / A)^{2}$ and power index $(\Delta P / P)$ lie close to a smooth curve.

Since the relation between these two quantities depends upon the distribution function of the amplitude of the radio wave, this result gives us some information about the distribution functions themselves. Any particular probability density function of amplitude should produce a single value of $(\Delta A / A)^{2}$ and a corresponding single value of $(\Delta P / P)$. The fact that the points on figure 9 lie near a single smooth curve implies that the family of probability density functions from which our scintillation samples have been taken can be described by a single parameter, the position along the line depending upon the value of that parameter.

The solid line in the figure shows the theoretical relation which would occur if the observed signal were composed of a steady (undiffracted) component and a random component consisting of a number (three or more) of independent, randomly phased, diffracted waves. The probability density function from which this curve has been derived was first described by Rice [1944 and 1945] and has been discussed in terms of its application to ionospheric problems by Ratcliffe [1956]. This probability density function, which we shall call the "Rice distribution", is really a family of distribution

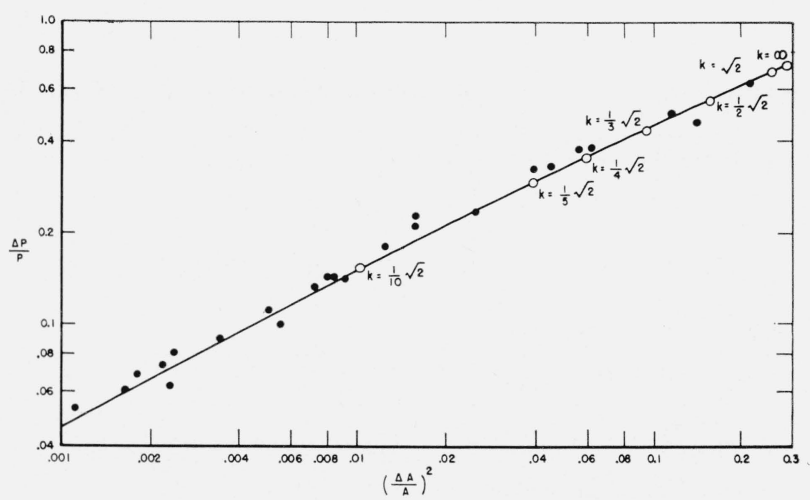

Figure 9. The relationship between amplitude and power indices, compared to the theoretical curve from the Rice distribution. 
functions parametric in the quantity

$$
\begin{aligned}
a & =\frac{\text { amplitude of the steady component }}{\text { rms instantaneous amplitude of the random component }} \\
& =\frac{\sqrt{2} \text { (rms instantaneous amplitude of the steady component })}{\text { rms instantaneous amplitude of the random component }} .
\end{aligned}
$$

Norton et al., [1955] have defined the quantity

$$
k^{2}=2 / a^{2}=\left[\frac{\mathrm{rms} \text { instantaneous amplitude of the random component }}{\mathrm{rms} \text { instantaneous amplitude of the steady component }}\right]^{2}
$$

which has the advantage of being a ratio of mean squares. $k$ may be thought of as the rms noise-tosignal ratio of the radio wave. In the present application "signal" means the steady component unaffected by the irregularities of the ionosphere, and "noise" means the combination of the various diffracted waves.

The Rice distribution degenerates into the familiar Rayleigh distribution in the limit as $k$ approaches infinity, and approaches a displaced Gaussian distribution as $k$ approaches zero. The details of the derivation of this curve have been given elsewhere [Lawrence and Jespersen, 1959].

The apparent agreement between the amplitude scintillation records and the Rice distribution means that the arbitrary amplitude and power indices may be replaced by the physically more meaningful parameter $k$. (This agreement is also justification for the method chosen to produce artificial test data, as described in the following paper.) Each depth index naturally covers a considerable range of values

\begin{tabular}{|c|c|c|c|}
\hline \multirow{2}{*}{ Depth index } & \multicolumn{3}{|c|}{$k$} \\
\hline & $\min$ & $\max$ & avg \\
\hline $\begin{array}{l}1 \\
2 \\
3 \\
4 \\
5\end{array}$ & $\begin{array}{r}0.04 \\
.05 \\
.13 \\
.28 \\
.47\end{array}$ & $\begin{array}{r}0.12 \\
.13 \\
.30 \\
.35 \\
>3\end{array}$ & $\begin{array}{r}0.05 \\
.07 \\
.20 \\
.31 \\
1.41\end{array}$ \\
\hline
\end{tabular}
of $k$. Extreme and average values are approximately as shown below.

The family of Rice distributions as defined by Rice [1944 and 1945] applies to the amplitude of the radio wave and is thus quite convenient for the study of communications signals. For radio-star observations it is usual to employ a square-law detector and thus obtain records which are linear in power. The Rice distributions, $G(P)$, plotted in terms of power, are shown in figure 10. These curves accurately match the observed distributions of chart deflections. On these curves the long tail which exists for large values of $k$ is particularly noticeable. The corresponding large deflections of the record are indeed prominent, and may easily give the erroneous impression that they are caused by some special mechanism unrelated to random diffraction.

Many simultaneous pairs of records at $53 \mathrm{Mc} / \mathrm{s}$ and $108 \mathrm{Mc} / \mathrm{s}$ have been used to compare the values of $(\Delta A / A)^{2}$ at the two frequencies. Booker [1958] has pointed out that, in the Fraunhofer region (i.e., in the far-zone region, where $(\Delta A / A)^{2}$ is independent of the distance between the observer and the diffracting screen $),(\Delta A / A)^{2}$ should vary as the square of the radiofrequency, while in the Fresnel region (i.e., the nearzone region) the variation should be as the fourth power of the frequency. Figure 11 seems to indicate that, for values of $(\Delta \dot{A} / A)^{2}$ less than about 0.2 at 53 $\mathrm{Mc} / \mathrm{s}$, we are on the border between these two regions. For large values (not shown in the fig.) $(\Delta A / A)^{2}$ approaches the Rayleigh limit $(0.274)$ independent of frequency [Lawrence and Jespersen, 1959]. This is expected, since the Rayleigh limit represents the case where all of the signal (undiffracted component) has been removed.

As has been indicated, the noise level may be estimated from the power spectra. This estimated level has been used to compute the contribution of noise to the value of $(\Delta A / A)^{2}$ as a function of $(\Delta A / A)^{2}$ at the two frequencies. The observed values of $(\Delta A / A)^{2}$ were then corrected for noise effects and these are the values plotted in figure 11 .

A rough indication of the diurnal variation, mixed with an unknown amount of seasonal variation, of amplitude scintillations comes directly from the subjective depth indices. The lower curve in figure 7 was drawn by smoothing the hourly average values of depth index, and converting the result to $(\Delta A / A)^{2}$

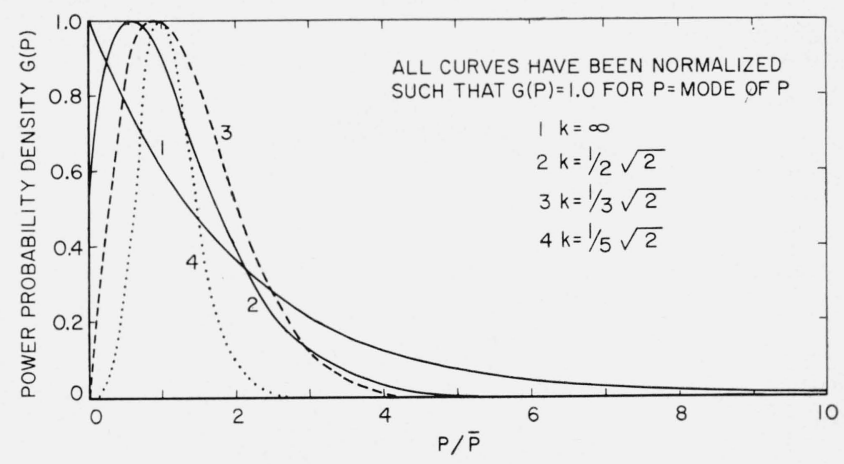

Figure 10. The Rice distribution function plotted in terms of power. 


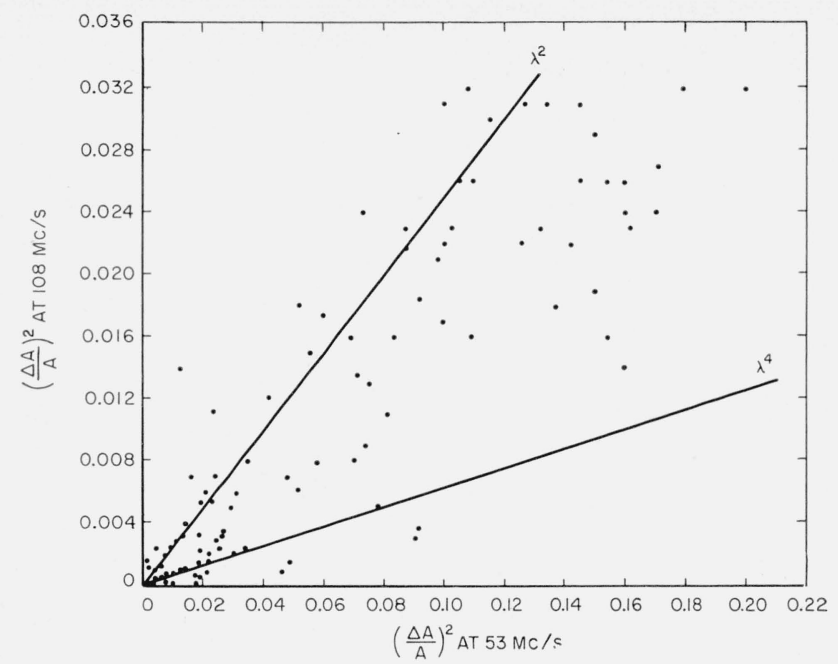

Figure 11. Amplitude scintillations at the two radiofrequencies. The expected wavelength dependence for Fraunhofer $\left(\lambda^{2}\right)$ and Fresnel $\left(\lambda^{4}\right)$ diffraction is shown by the solid lines.

by means of the table (see appendix 1). These curves are certainly too low because of a limitation at the high end of the scale of depth index. Index 5 has been used to describe records in which the scintillations were "so deep that the lobes disappeared". Since many different degrees of scintillation activity are included in this category, the effects of the most violent scintillations are not properly represented in the lower curves of figure 7 . In order to assess the importance of this saturation effect, a portion of the data has been analyzed by the more elaborate procedure of evaluating $P_{n}$ (see following paper). 'The results, when transformed to equivalent values of $(\Delta A / A)^{2}$ and then averaged by months, are shown by the upper curves in figure 7 . These curves give a more accurate estimate of the true variation of $(\Delta A / A)^{2}$. The data used in this result were a composite of two groups centered around the elevation angles $15^{\circ}$ and $35^{\circ}$.

Figure 12 shows the elevation-angle dependence of $(\Delta A / A)^{2}$ as computed from $P_{n}$. Only data from August 1958 to February 1959 are involved here. For comparison, a curve proportional to $\sec ^{3} \chi$, the cube of the secant of the zenith angle, is included on the same figure. The observed variation does not agree very well with the $\sec ^{3} \chi$ curve which is predicted by a theory based upon plane-earth geometry and spherical irregularities [Booker, 1958]. However, most observers agree that the irregularities are alined along the earth's magnetic field and are therefore not spherical. Also at the elevations considered, the curvature of the earth is not negligible. Perhaps the most important reason for the discrepancy is that $(\Delta A / A)^{2}$ approaches some constant value independent of frequency, and hence, for large scintillations, any theoretical elevation-angle dependence should be modified to include this effect.

Using the noise-to-signal ratio, $k$, which indicates the fraction of incident energy that suffered diffraction in the ionosphere, we can make a rough compari-

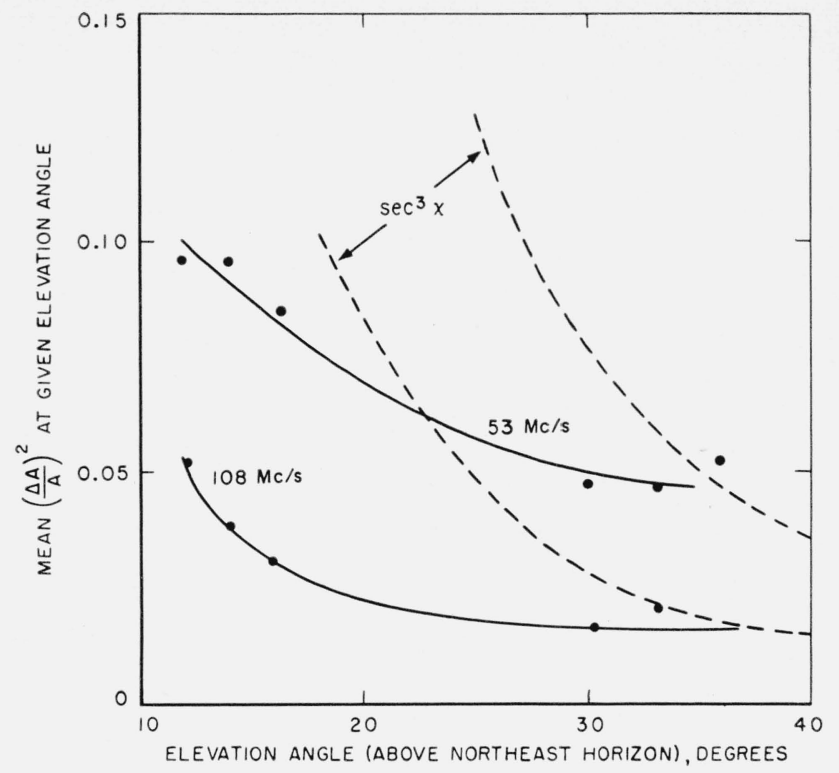

FIGURE 12. The variation, with source elevation above the northeastern horizon, of the amplitude index for each radiofrequency.

The broken lines show a theoretical elevation angle dependence proportional to the cube of the secant of the zenith angle.

son of our results with those of other observers. Interpolating between our two frequencies, we find that, for a given elevation angle, the value of $k$ observed at Boulder is 2 or 3 times greater than the value observed at $80 \mathrm{Mc} / \mathrm{s}$ at Cambridge, England [Hewish, 1952]. Since measurements at College, Alaska [Little et al., 1958] have shown little or no dependence upon zenith angle, direct comparison with the Boulder results is less meaningful. The value of $k$ at College was consistently 2 or 3 times greater than that at Boulder, even near the horizon, despite the fact that the College measurements were made at $223 \mathrm{Mc} / \mathrm{s}$. (The Boulder and College observations were made when the relative sunspot number was about 190 ; for the Cambridge observations it was 85.)

Angular scintullations. When normalized to account for the spacing of the interferometer lobes, the phase plots can be used to calculate power spectra of angular scintillations over the frequency range 0.005 to $0.17 \mathrm{c} / \mathrm{s}$. Figure 13 shows examples of such spectra. The ordinate value is the common logarithm of power density, or variance per unit frequency interval, in units of tenths of a minute of arc, squared. Here, as with the corresponding spectra of amplitude scintillations, the reader is reminded that spectra can be obtained only for moderately disturbed records. Records containing intense scintillation activity cannot be used to derive spectra.

Hewish [1951] has shown that for meter wavelengths, the scale of the ionospheric irregularities is the same as the scale of the phase irregularities on the ground whenever the rms phase variations are less than 1 radian. That this is generally true for our data is shown by the fact that the shape of the power spectra of the angular scintillations are similar for 


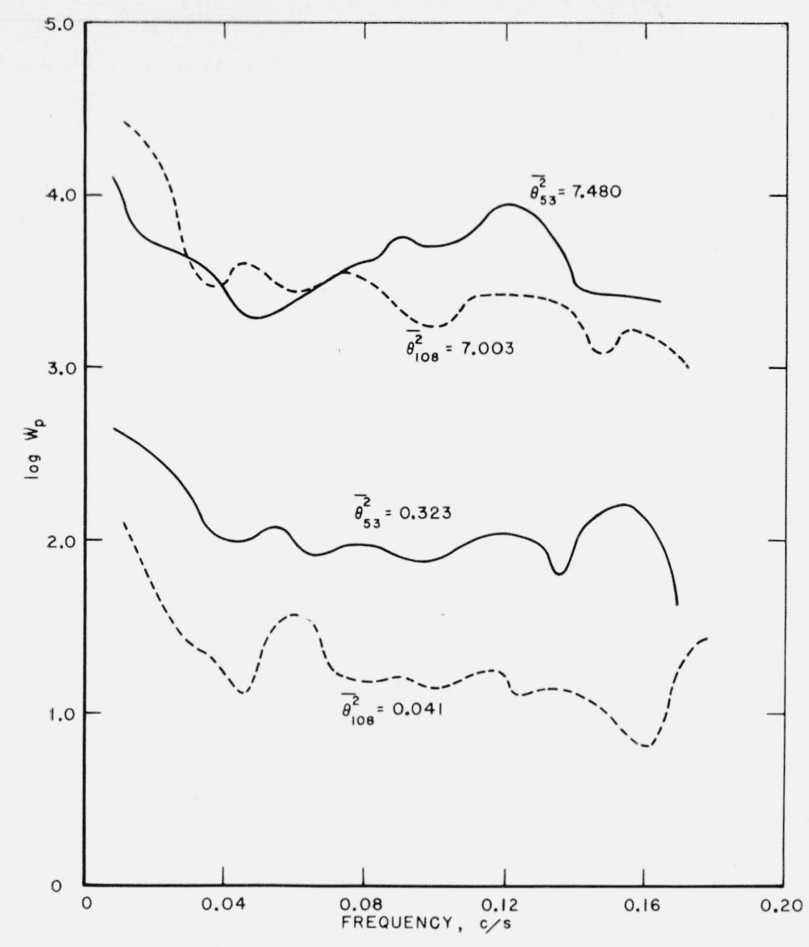

FIGURE 13. The highest and lowest observed angular power spectra for each radiofrequency.

The lower level at each frequeney indicates the background "noise" level. $W_{p}$ is the variance per unit frequency interval expressed in tenths of a minute of arc squared. $\overline{\theta^{2}}$ is indicated, in units of minutes of arc squared.

records taken simultaneously at the two radiofrequencies. However, as in the case of amplitude scintillations, the high-frequency end of the power spectra is at times masked by the presence of noise which is folded back into the spectrum. Figure 13 shows the highest and lowest observed levels of the power spectra for angular scintillations at the two frequencies. As for amplitude scintillations, the lower two curves are indicative of the noise level. The corresponding values of the variance of the angular scintillations, $\overline{\theta^{2}}$, are given for each of the spectra.

The shape of the spectrum varies sufficiently from day to day to obscure any variation in spectral shape which may be related to the elevation angle of the source. Inspection of simultaneous power spectra of angular scintillations at 53 and $108 \mathrm{Mc} / \mathrm{s}$ indicates that the details of the spectra do not coincide. The general shape may vary from hour to hour, usually in the same way on both radiofrequencies.

Inspection of the phase plots of records taken simultaneously at the two radiofrequencies indicates (except for a special case to be described) that there is no detailed correlation. Here again, where diffraction is involved, no correlation is expected.

Forty-one pairs of simultaneous phase plots (representing moderate scintillation activity) were used to compare $\overline{\theta^{2}}$, the mean square angular scintillation at the two frequencies. Figure 14 displays this frequency dependence of angular scintillations.

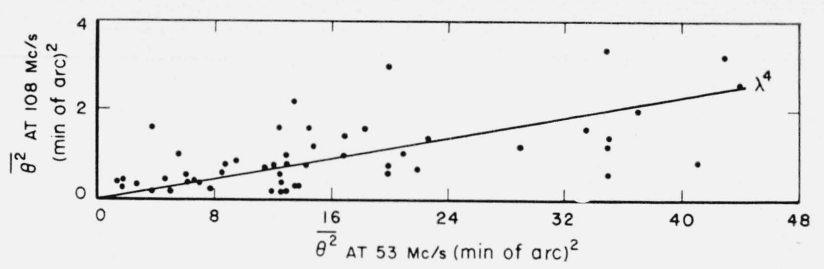

Figure 14. Mean square angular scintillation at the two radiofrequencies.

The solid line is the theoretical wavelength dependence.

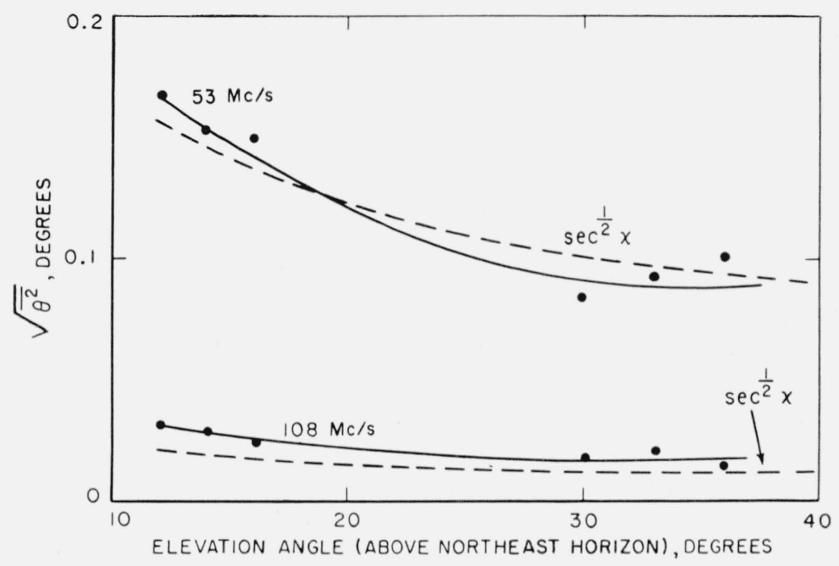

FIGURE 15. The variation, with source elevation above the northeastern horizon, of the rms angular scintillation for each radiofrequency.

The broken lines show a theoretical elevation-angle dependence proportional to the square root of the secant of the zenith angle.

Theory [Booker, 1958] indicates that there should be a fourth-power wavelength dependence for $\overline{\theta^{2}}$, and our data fits this quite well, Here there is no tendency to approach a limiting value which is independent of frequency, since $\overline{\theta^{2}}$ depends upon the number of ionospheric irregularities which the radio wave passes through, each irregularity introducing a certain phase change dependent upon the frequency of the incident wave.

Figure 15 shows the elevation-angle dependence of $\overline{\theta^{2}}$ at the two frequencies and figure 16 gives the seasonal (and/or diurnal) dependence of $\overline{\theta^{2}}$ over a limited portion of the year. Both of these curves are based upon $\sigma_{d}$ (see following paper by the same authors) which was then transformed to the appropriate value of $\overline{\theta^{2}}$ Simple theory indicates that $\overline{\theta^{2}}$ should vary as $\sec ^{1 / 2} \chi$ and this seems to be true in figure 15 .

Relationships between amplitude and angular scintillations. About two hundred scatter diagrams of the instantaneous power, $P$, versus the instantaneous phase, $\alpha$, have been examined. In no instance is there an indication of a detailed correlation between these two quantities. This is the result to be expected if a diffraction process is involved. However, a definite correlation does appear for the magnitude of power and phase variations averaged over $10 \mathrm{~min}$ intervals. 


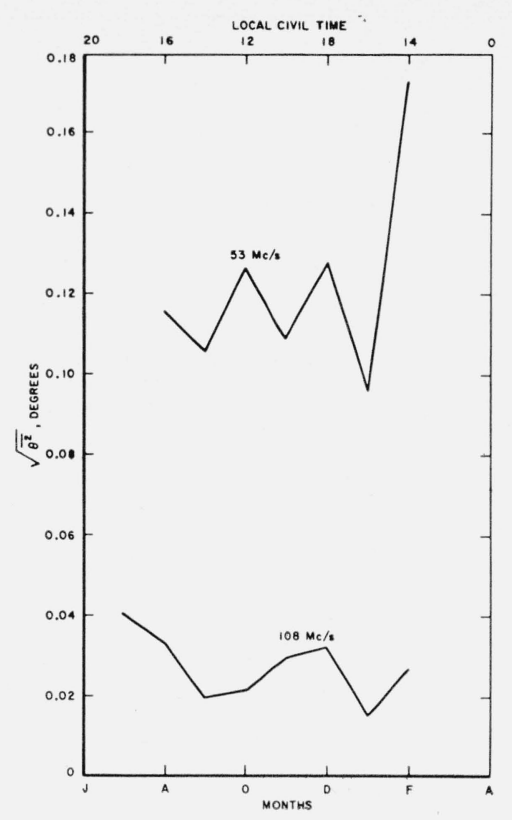

Figure 16. The seasonal and/or diurnal variation of $\mathrm{rms}$ angular scintillation.

Theory [Booker, 1958] indicates that the statistical relation between amplitude and angular scintillations depends upon the observer's distance from the ionospheric disturbing region. In the Fraunhofer region

$$
\left(\frac{\Delta A}{A}\right)^{2}=\frac{1}{2}\left(\frac{2 \pi L}{\lambda}\right)^{2} \overline{\theta^{2}}
$$

whereas in the Fresnel region

$$
\left(\frac{\Delta A}{A}\right)^{2}=\frac{z^{2}}{8(\pi L)^{2}} \overline{\theta^{2}}
$$

$L$ is the ionospheric irregularity size and $z$ is the observer's distance from the disturbing region. These results are valid only for rms phase scintillations less than 1 radian. From (1) it is evident that the relation between $(\Delta A / A)^{2}$ and $\overline{\theta^{2}}$ is linear, with slope proportional to $(L / \lambda)^{2}$ in the Fraunhofer region. In the Fresnel region the relation depends upon $z$, the observer's distance from the disturbing region, and, furthermore, a plot of $(\Delta A / A)^{2}$ versus $\overline{\theta^{2}}$ should coincide for two different radiofrequencies.

Figure 17 shows the relation which was obtained by fitting a line through the observed scatter of points. Because $(\Delta A / A)^{2}$ increases with zenith angle, the horizontal scale corresponds to a nonlinear scale of increasing $z$. The curves diverge, indicating a dependence on $\lambda$, but they are also nonlinear, indicating a dependence on $z$. We must conclude that our observations were not completely within either the Fraunhofer or the Fresnel regions, but in the transition zone.

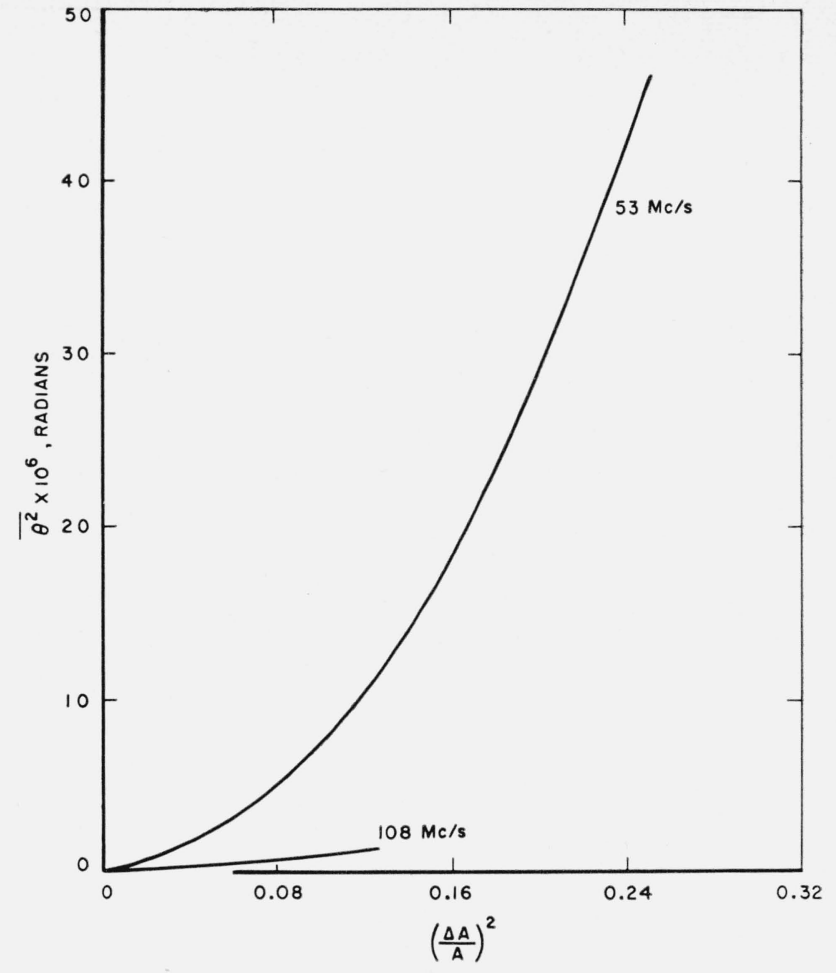

FIGURE 17. The observed relationship between mean square angular scintillation and amplitude index.

Examination of the spectra of the power and phase scintillations from a given record indicates that there is no detailed correlation between the two, but that they have the same general shape, viz, steep at the low-frequency end, and leveling off toward higher frequencies. This is in agreement with other observations that the rate of fading of the amplitude and phase are similar for a given record [Ryle and Hewish, 1950].

Slow, irregular angular fluctuations. In addition to the angular scintillations described above, slow irregular phase variations having autocorrelation times of some minutes have been observed at both frequencies. Figure 18 shows two typical examples of these irregularities as observed simultaneously at the two radiofrequencies. If one were to remove the slow variation from them they would look like ordinary scintillation records in which no irregularities are present. Figure 19 shows one of the slow irregular structures measured for a period of $1 \mathrm{hr}$. This figure is a composite of consecutive records of the type shown in figure 18. It should be noted that the steady angular variation of the radio star, due to its diurnal motion, has been removed, as it is from all phase plots. Since no attempt has been made to determine the absolute phase of the interferometer system, the zero levels are arbitrary on these diagrams.

The slow angular variations show high correlation between records taken simultaneously at the two frequencies. Forty-seven pairs of records, each ten 

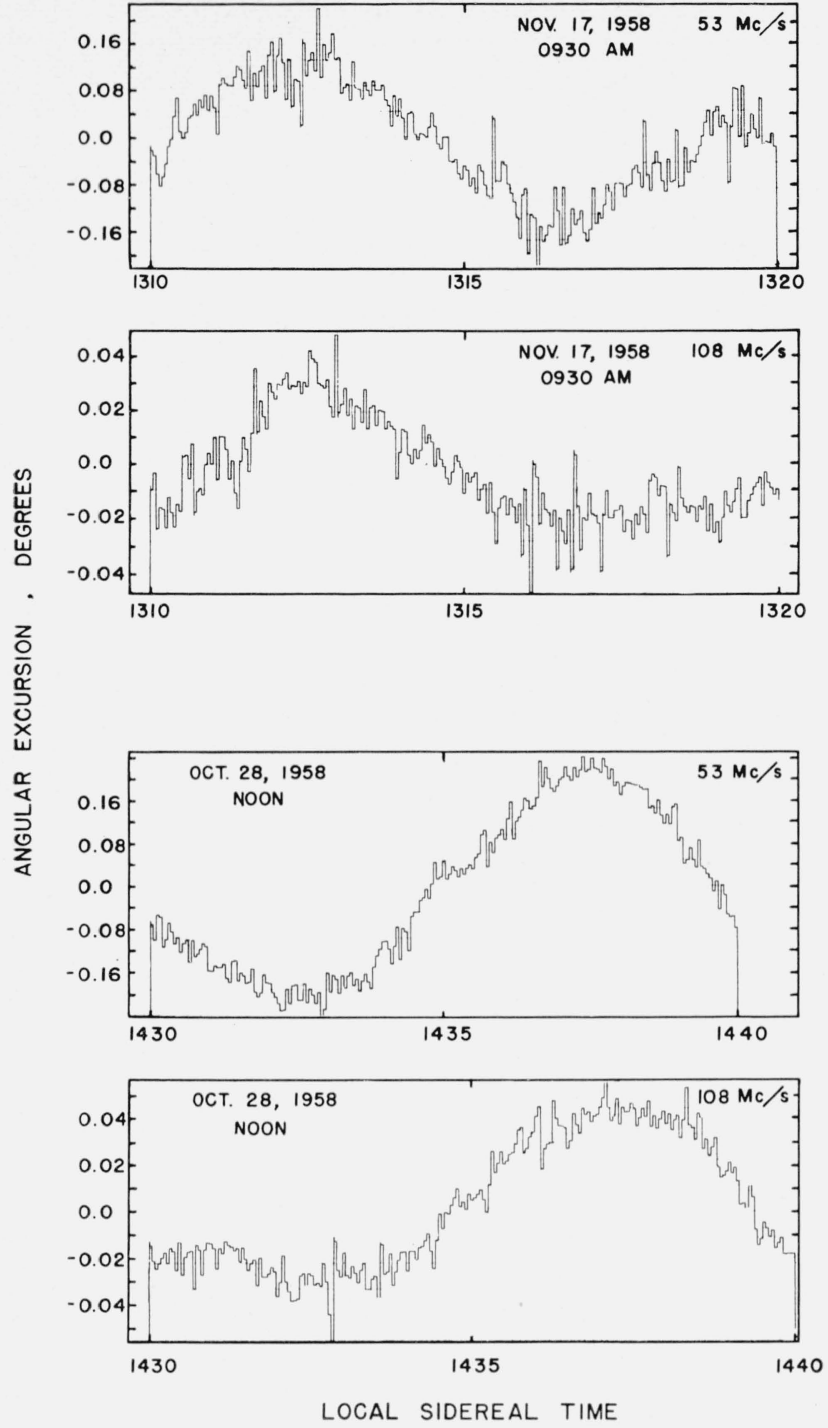

FIgURE 18. Typical examples of slow irregular angular variation observed simultaneously at the two radiofrequencies.

ELEVATION OF CYGNUS-A ABOVE NORTHEAST HORIZON, DEGREES

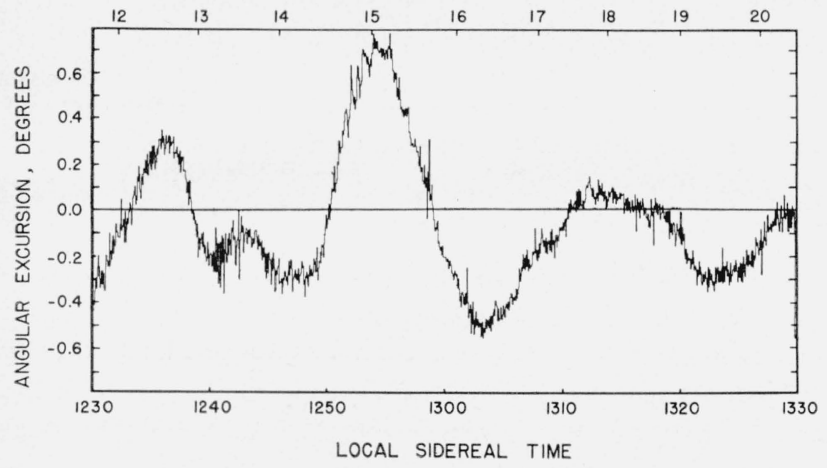

FIGURE 19. A sixty minute record exhibiting slow irregular angular variation.

$108 \mathrm{Mc} / \mathrm{s} ; 1100$ a.m., October 17, 1958 minutes long, showing slow irregular variations at the two frequencies, have been examined to determine the average total angular excursion for each frequency. The ratio of the average excursion at $53 \mathrm{Mc} / \mathrm{s}$ to that at $108 \mathrm{Mc} / \mathrm{s}$ ranges from $2: 1$ to $6: 1$ with the average 4.1:1. A four-to-one ratio would be expected if ionospheric refraction were responsible for the angular excursions. The close agreement between the observed ratio and the theoretical ratio expected for refraction, together with the high cross-correlation, indicates that these slow angular variations are due to ionospheric refraction. Tests with a target transmitter were made to eliminate the possibility that equipment malfunction might be responsible for these slow angular variations. The negative results of these tests, together with the frequency dependence of the variations, indicate that the effects are of ionospheric origin.

Examination of the amplitude variations of pairs of records for which there were slow angular scintillations, indicates that there is no correlation between the amplitude fluctuations on the two frequencies, nor is there any long-period variation in amplitude. In fact, the amplitude scintillations seem to be unaffected by the presence of the slow angular scintillations.

This lack of correlation suggests that the ionospheric irregularities responsible for the refraction are acting as prisms, or as weak lenses whose focal lengths are long compared with the observer's distance from them. For the case of a prism we can use the observed total angular excursion to estimate the amount by which the electron-content of the ionosphere deviates from its mean value. Smith [1952] has shown that a prism will introduce an excursion in apparent direction given by

$$
\Delta \theta=\frac{e^{2}}{2 \pi m f^{2}} \frac{d n}{d x}
$$

Here $d n / d x$ is the gradient, transverse to the line of sight, in electron content per unit column along the line of sight; $f$ is the radiofrequency; $e$ is the charge of an electron; and $m$ is the mass of an electron.

A typical angular excursion at $53 \mathrm{Mc} / \mathrm{s}$ is about 0.5 deg. If we assume that such a gradient is maintained over a portion of the irregularity equal to, say, $10 \mathrm{~km}$, then from the above formula, the total electron-content variation is a few percent of the mean value.

Figure 20 shows the seasonal and/or diurnal variation in the frequency of occurrence of these irregularities. The ordinate value is the percent of observations which exhibit slow irregular variations, relative to the total number of cases available for each month. The shape of the curve suggests that the variation is more diurnal than seasonal, since the peak is centered about two seasons (fall and winter). Furthermore, in the months from May to December the data were taken during daylight hours. The shape of the curve seems to indicate that the irregularities build up rapidly as the ionosphere becomes illuminated, and then gradually disappear. 


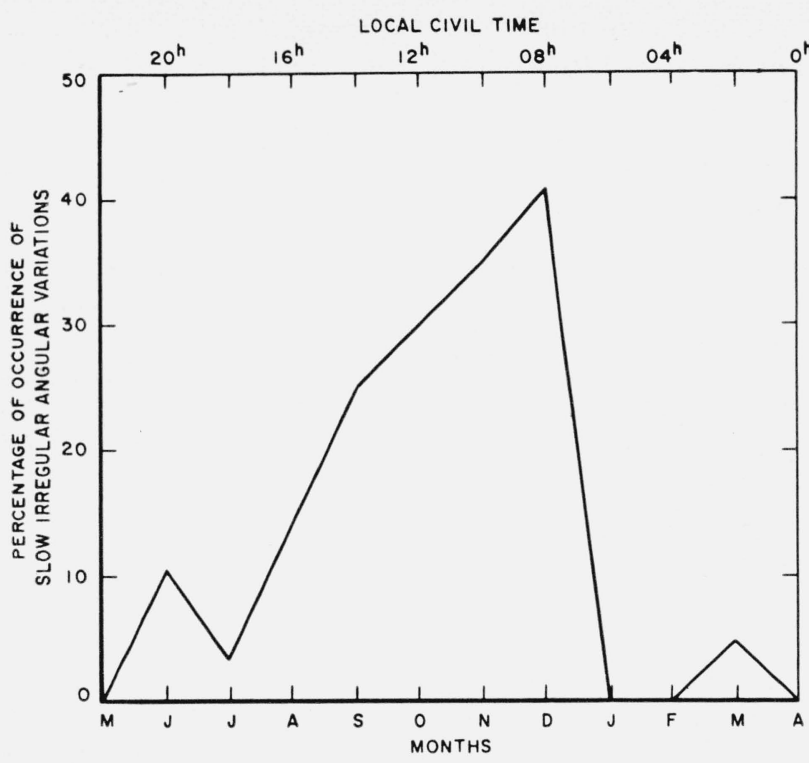

Figure 20. The seasonal and/or diurnal varnation in the frequency of occurrence of slow irregular angular variations.

The total angular excursion is found to decrease as the source rises, while the probability of observing these slow irregularities seems to increase. Since ordinary amplitude and phase scintillations decrease as the source rises, the slow irregularities are most likely to be seen on records for which the ordinary scintillations are small, $(\triangle A / A)^{2}$ less than 0.06 . However, they have occurred on several occasions when $(\Delta A / A)^{2}$ was as large as 0.20 .

It is reasonable to assume that the elevationangle dependence in the probability of occurrence of the slow angular variations results from the fact that at low elevation angles amplitude scintillations are most severe and are likely to limit the ability of the "zero-crossing" method to recover the slow phase variations from the data.

The variation in the total angular excursion with elevation angle probably arises from the changing path length through the ionosphere, although there is some possibility that the structure of the irregularities varies rapidly with latitude. No attempt has yet been made to check these ideas quantitatively.

At the low elevation angles of 10 to $20 \mathrm{deg}$, the distance which the line of sight moves through the ionosphere in a period of $20 \mathrm{~min}$ (typical of the length of time during which any one irregularity is observed) is of the order of $200 \mathrm{~km}$. This distance may be assumed to be indicative of the horizontal size of the ionospheric irregularities in the appropriate direction if ionospheric drifts are neglected.

Examination of the distributions of ionospheric parameters scaled from the Ellsworth ionograms reveals no significant difference between times of slow angular scintillations and other times. A possible exception is a marginally significant increase in $f_{0} F 2$.
Vitkevich and Kokurin [1957 and 1958], using a radioastronomy technique, observed large-scale angular variations with no corresponding amplitude effects. Other authors have apparently observed a similar phenomenon [Bramley and Ross, 1951; Whitehead, 1956; Jones et al., 1957]. The large-scale traveling disturbances which Munro [1950] has studied are possibly related to the disturbances observed here. Satellite observations made at the Boulder Laboratories of the National Bureau of Standards [Little and Lawrence, 1960] have also disclosed the presence of these large-scale, ionospheric irregularities.

\section{Conclusions}

After removal of the diurnal (and/or seasonal) trends from the data, amplitude scintillations still correlate with spread $F$. The variation of correlation with elevation angle of the source is small, indicating that spread $F$ is in patches whose sizes are at least $400 \mathrm{~km}$. No significant correlation is found between amplitude scintillations and sporadic $E$. This result is particularly interesting since the experiment was designed especially to measure and detect possible $E$-region effects on scintillations.

The distribution of flux received from a scintillating radio source closely matches the theoretical distribution to be expected from the superposition of several randomly phased, diffracted waves upon a constant, undiffracted wave. The zenith-angle dependence of these amplitude scintillations does not agree with a theory based upon isotropic ionospheric inhomogeneities. At Boulder, the fraction of the radio-star energy diffracted by the ionosphere is about twice as great as at Cambridge, England, but less than half as great as at College, Alaska. However, the Boulder and College observations were made when the relative sunspot number was about 190 ; for the Cambridge observations it was 85 .

The root-mean-square value of angular scintillations is found to be proportional to the square of the wavelength, in accord with a theory of diffraction by ionospheric irregularities.

Comparison of amplitude scintillations with angular scintillations indicates that, for elevation angles of $15^{\circ}$ to $50^{\circ}$, the region of the ionosphere responsible for scintillations lies near the border between the Fresnel and Fraunhofer diffraction regions for both freauencies.

Slow irregular angular variations having autocorrelation times of 5 to $20 \mathrm{~min}$ are commonly observed at both frequencies. These variations occur chiefly by day and are attributed to large (of the order of $200 \mathrm{~km}$ ) lens-like ionospheric irregularities whose electron content per unit vertical column varies by a few percent from the mean value. The resulting angular disrlacement of a radio source is frequently $0.5 \mathrm{deg}$ at $53 \mathrm{Mc} / \mathrm{s}$, and varies as the square of the wavelength.

\section{Appendix 1}

Amplitude scintillation index. A visual estimate of the depth of scintillations can be made from a penrecorder trace of the interferometer output. Figure 
21 shows 12 -min sections of two typical scintillation records. The modulated sine wave is formed as the interferometer lobes sweep past the discrete source (Cygnus-A) at the rate of $10 / \mathrm{min}$. The amplitude of the sinusoidal trace is proportional to the flux received from the discrete source; the fluctuations in the envelope are ionospheric scintillations. The depth of these amplitude scintillations was classified by eye into 5 categories as follows:

\begin{tabular}{|c|c|}
\hline $\begin{array}{l}\text { Depth } \\
\text { index }\end{array}$ & Appearance of record \\
\hline $\begin{array}{l}1 \\
2 \\
3 \\
4 \\
5\end{array}$ & $\begin{array}{l}\text { No scintillations. } \\
\text { Shallow scintillations. } \\
\text { Medium scintillations. } \\
\text { Deep scintillations. } \\
\text { Scintillations so deep that } \\
\text { the lobes disappear. }\end{array}$ \\
\hline
\end{tabular}

For example, the records in figures $21 \mathrm{a}$ and $21 \mathrm{~b}$ have been assigned depth indices 3 and 5 respectively.
The estimated depth index for each 5 -min period was inserted upon the appropriate IBM card.

Many of the scintillation records were supplemented by simultaneous digital recordings on punched paper tape. A random sample of $108 \mathrm{Mc} / \mathrm{s}$ digital records was selected for each depth index and subjected to detailed analysis on an electronic computer. This analysis yielded both the power index

$$
\frac{\Delta P}{P} \equiv \overline{\frac{P-\bar{P}}{\bar{P}} \mid}
$$

and the amplitude index

$$
\left(\frac{\Delta A}{A}\right)^{2} \equiv \overline{\left(\frac{A-\bar{A}}{\bar{A}}\right)^{2}}
$$

where $P$ is the power received from the discrete source, $A=\sqrt{P}$ is the amplitude of this radio signal, and a bar denotes an average over the record. The

\begin{tabular}{|c|c|c|c|c|c|c|}
\hline \multirow{2}{*}{$\begin{array}{l}\text { Depth } \\
\text { index }\end{array}$} & \multicolumn{3}{|c|}{$\Delta P / P$} & \multicolumn{3}{|c|}{$(\Delta A / A)^{2}$} \\
\hline & $\min$ & $\max$ & $\operatorname{avg}$ & $\min$ & $\max$ & avg \\
\hline $\begin{array}{l}1 \\
2 \\
3 \\
4 \\
5\end{array}$ & $\begin{array}{r}0.04 \\
.06 \\
.14 \\
.30 \\
.44\end{array}$ & $\begin{array}{r}0.13 \\
.14 \\
.35 \\
.39 \\
.75\end{array}$ & $\begin{array}{r}0.09 \\
.10 \\
.23 \\
.35 \\
.57\end{array}$ & $\begin{array}{r}0.0008 \\
.0016 \\
.0092 \\
.0377 \\
.0838\end{array}$ & $\begin{array}{r}0.0071 \\
.0083 \\
.0448 \\
.0575 \\
.3237\end{array}$ & $\begin{array}{r}0.0025 \\
.0045 \\
.0238 \\
.0512 \\
.1689\end{array}$ \\
\hline
\end{tabular}
results are summarized in the following table:
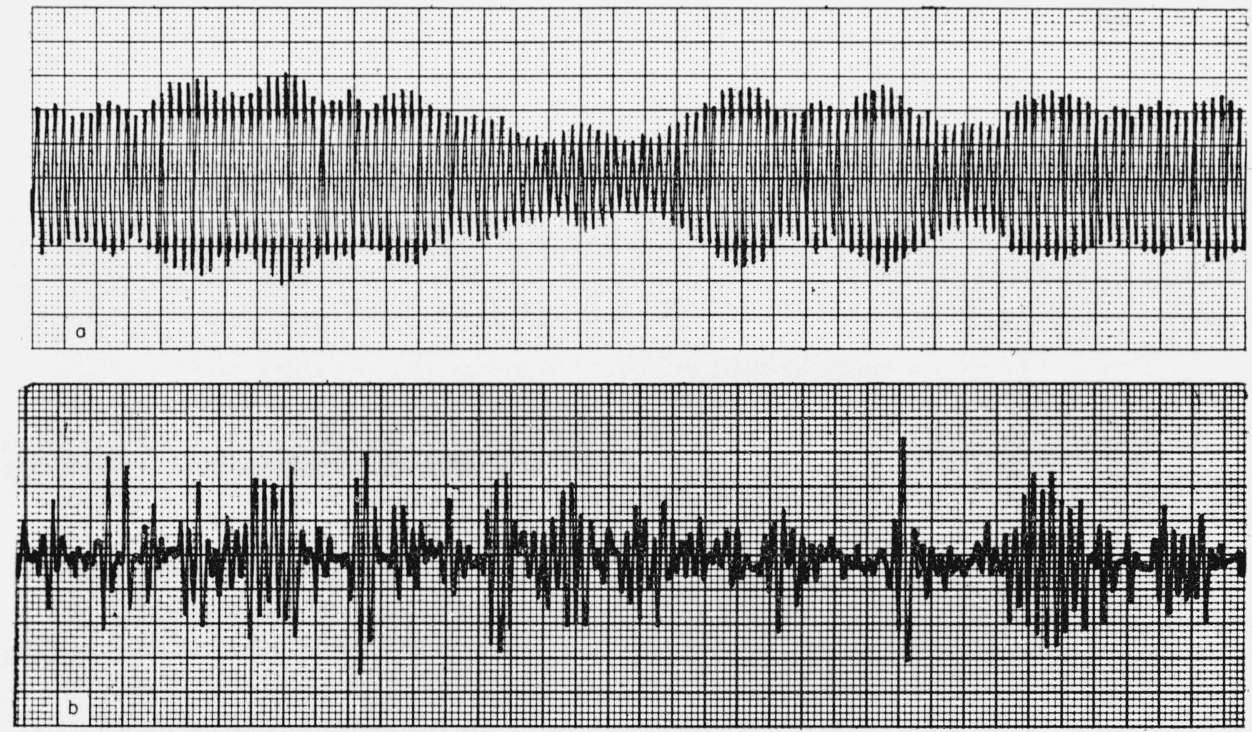

Figure 21. Twelve-minute samples of typical $108 \mathrm{Mc} / \mathrm{s}$ phase-sweeping records.

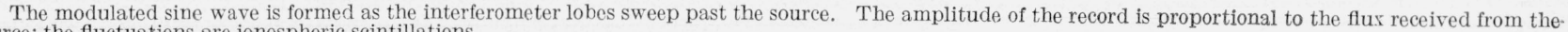
source; the fluctuations are ionospheric scintillations. 


\section{Appendix 2}

Correlation of amplitude indices with ionograms. The IBM cards containing the simultaneous ionospheric (Ellsworth) and scintillation data are referred to as "statistical index cards". Tests for correlation between any two parameters, $x$ and $y$, on the cards may be performed easily on IBM statistical machines. The statistical-sorter output consists of a bivariate distribution table, each variable, $x$ and $y$, being allowed a scale of one through nine, zero being reserved for cases where there are no data. These ten-by-ten distribution arrays are then punched onto IBM cards for processing through a computer program which determines the correlation coefficient between the two variables. The normalized correlation coefficient is defined in the usual manner as

$$
r=\frac{\overline{x y}-\bar{x} \bar{y}}{\sigma_{x} \sigma_{y}}
$$

where the variance $\sigma_{x}^{2}=\bar{x}^{2}-\bar{x}^{2}$. For independent data which are normally distributed, the standard deviation of the correlation coefficient is

$$
\sigma_{r}=\frac{1-r^{2}}{\sqrt{ } N}
$$

where $N$ is the number of samples. If the data are not independent, but possess a significant serial correlation over an interval $n$ samples in length, then $N$ in (2) should be replaced by $N / n$. A visual inspection of the data indicates $n$ to be approximately 6 for data taken at 5 min intervals, but nearly 1 for data taken at half-hour intervals. For the correlation coefficients reported here, the standard deviations have been calculated using these values of $n$.

The distribution of the data is approximately normal for most of the ionospheric parameters but for scintillations it is strongly skewed towards the high depth indices. Figure 22 gives the distribution of scintillation index for each radiofrequency, both before and after revision of the data. The methods of, and reasons for, data revision will be discussed in the following paragraphs. In order to assess the error introduced by using (2) with skewed data, a more sophisticated analysis [Cramer, 1954] was undertaken. This calculation, involving higher order moments of the actual distribution, was made on a typical correlation coefficient taken from our data. The difference in $\sigma_{r}$ amounted to less than 0.001 ; hence (2) has been used in all subsequent calculations of $\sigma_{r}$.

In cases where $|r|>0.5$, or where $N$ is small, there is some advantage to use of the Fisher $Z_{r}$ function [Walker and Lev, 1953] and its corresponding standard deviation $\sigma_{z_{r}} \cdot Z_{r}$ is defined as

$$
Z_{r} \equiv \frac{1}{2} \ln \left(\frac{1+r}{1-r}\right)
$$

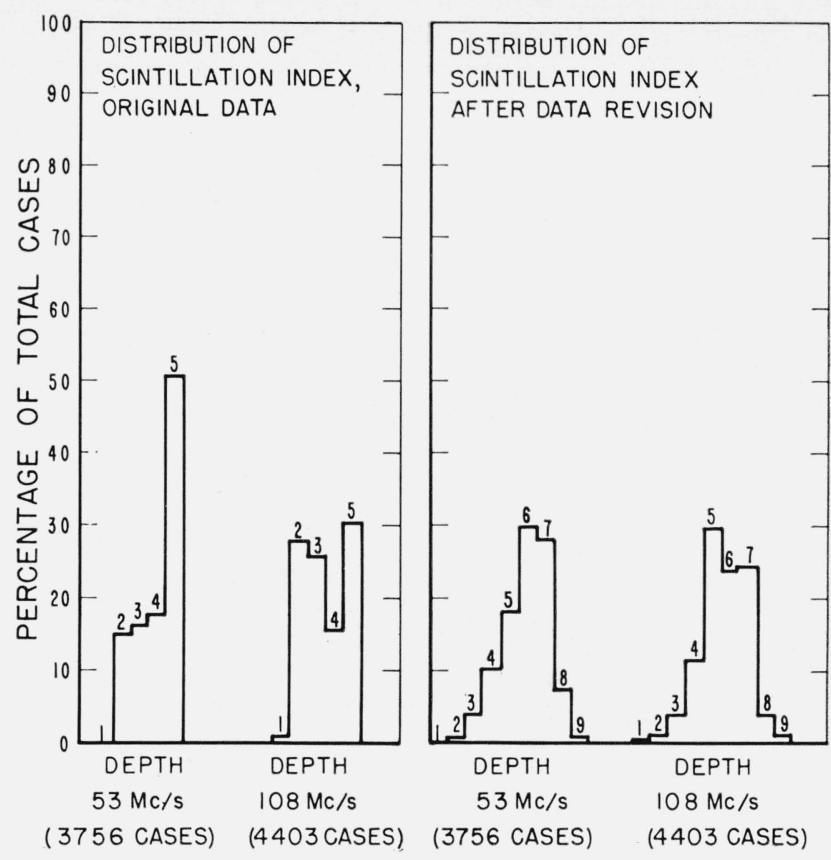

FIGURE 22. Frequency distribution of observed scintillation index.

and $\sigma_{z_{r}}$ is obtained from the formula

$$
\sigma_{z_{r}}=\frac{1}{\sqrt{ } N-3} .
$$

In the present case where correlation is small and $N$ is large, $Z_{r}$ and $\sigma_{z_{r}}$ are practically equal to $r$ and $\sigma_{r}$ respectively, and they can be used interchangeably.

The geometry of the experiment indicates that any correlation between ionospheric and scintillation parameters should vary with local sidereal time (LST) as the ray path from Cygnus-A moves through the different effective ionospheric levels over Ellsworth. Also, scintillation activity is found to decrease with increasing elevation angle (increasing LST) of the source. Consequently, the statistical index cards were divided into groups according to the elevation angle of Cygnus-A. Figure 3 gives these groupings as well is a complete distribution table of the cards as a function of the elevation angle of Cygnus-A. Figure 3 also gives the relationship between the elevation angle of Cygnus-A, local sidereal time (LST), and effective ionospheric heights. The average elevation angle of each major $\left(10^{\circ}\right)$ group has been computed and is used for plotting the correlation coefficients in section 5 .

Early correlation studies, reported previously [Lawrence and Jespersen, 1959], consisted primarily of bivariate correlations as a function of LST, and used only six months' data. With the cessation of recording, the thirteen months (February 1958 through February 1959) of available data were placed on statistical index cards. These data were then tested for correlations, but with few consistent results. By contrast, the previously reported 
results [Lawrence and Jespersen, 1959] of correlation of a portion of the data had appeared internally consistent. Prominent diurnal and/or seasonal effects in the full year's data are presumably responsible for this difference.

Division of the data into two classes, sunlit and dark ionosphere, proved helpful and indicated that complete removal of diurnal and seasonal trends would be worthwhile. To determine the trends, the hourly average value of each variable was plotted, except that in the case of spread $F$ and sporadic $E$ the frequency of occurrence was used, as shown in figure 6 . The hourly average values of scintillation depth index, converted to $(\Delta A / A)^{2}$, appear in figure 7 . These graphs are referred to as diurnal curves, although each contains an unknown amount of seasonal variation. It is impossible to distinguish with certainty between diurnal and seasonal effects, since only one radio source was used.

The IBM 650 computer was used to subtract the appropriate hourly average from each numerical value on the statistical index cards, and to normalize the remainder to a scale of one through nine. The resulting cards are referred to as "revised statistical index cards", and have been used in all the correlation results reported here. Figure 22 shows the effect of this revision upon the distribution of the scintillation indices. This redistribution cannot, of course, remove the effect of skewness in the original depth indices, as this was a result of limited equipment sensitivity.

The data in final form consist of several thousand revised statistical index cards. The extreme excursions of the inospheric and scintillation indices from their average value for any particular local time, now appear as extreme values on a scale of one through nine. Most of the data are clustered about the median of the scale and so they contribute little to the correlation coefficient. Thus the revised values are in a form which may be tested for detailed correlation between individual events, regardless of trends in the averages of the original variables.

This experiment could not have been performed without the cooperation of several people. Among them are E. R. Schiffmacher and H. A. Erickson who were responsible for the maintenance and operation of the scintillation receivers, R. F. Carle who established and operated the ionosonde at Ellsworth, and Mrs. C. I. Enfield who assisted with the analysis of the data. This project was supported by the Ballistic Missiles Division of the United States Air Force under D. O. AF 04(647)-134.

\section{References}

Blackman, R. B. and J. W. Tukey, The measurement of power spectra, Dover Publications, Inc., p. 32 (1959).

Bolton, J. G., O. B. Slee, and G. J. Stanley, Galactic radiation at radio frequencies VI; low-altitude scintillations of the discrete sources, Aust. J. Phys. 6, 434-451 (1953).

Booker, H. G., The use of radio stars to study irregular refraction of radio waves in the ionosphere, Proc. IRE 46, 298-314 (1958).
Bramley, E. N. and W. Ross, Measurements of the direction of arrival of short radio waves reflected at the ionosphere, Proc. Roy. Soc. A, London 207, 251-267 (1951).

Briggs, B. H., A study of the ionospheric irregularities which cause spread-F echoes and scintillations of radio stars, J. Atm. Terr. Phys. 12, 34-45 (1958).

Cramer, H., Mathematical methods of statistics, Princeton U. Press, p. 359 (1954).

Dagg, M., Diurnal variations of radio-star scintillations, spread F and geomagnetic activity, J. Atm. Terr. Phys. 10, 204-214 (1957).

Dueño, B., Study and interpretation of low angle fluctuations from the radio star Cassiopeia as observed at Ithaca, N.Y., Tech. Rept. No. 27, Cornell U. School of Elec. Eng., Ithaca, N.Y. (1955).

Dyce, R. B., VHF auroral and sporadic E propagation from Cedar Rapids, Iowa to Ithaca, N.Y., Trans. IRE AP-3, 76-80 (1955)

Hartz, T. R., Solar-terrestrial relations, Radio Physies Lab., Ottawa, Report 23-2-3 (1958)

Hewish, A., The diffraction of radio waves in passing through a phase-changing ionosphere, Proc. Roy. Soc. A, London, 209, 81-96 (1951).

Hewish, A., The diffraction of galactic radio waves as a method of investigating the irregular structure of the ionosphere, Proc. Roy. Soc. A, London, 214, 494-514 (1952)

Jones, J. L., B. Landmark and C. S. G. K. Getty, Movements of ionospheric irregularities observed simultaneously by different methods, J. Atm. Terr. Phys. 10, 296-301 (1957).

Koster, J. R., Radio star scintillations at an equatorial station, J. Atm. Terr. Phys. 12, 100-109 (1958).

Lawrence, R. S., An investigation of the perturbations imposed upon radio waves penetrating the ionosphere, Proc. IRE 46, 315-320 (1958)

Lawrence, R. S. and J. L. Jespersen, A preliminary analysis of amplitude scintillations of radio stars observed at Boulder, Colorado, Tech. Note No. 20, NBS Boulder Labs. (1959)

Little, C. G. and A. Maxwell, Fluctuations in the intensity of radio waves from galactic sources, Phil. Mag., Ser. $7 \mathbf{4 2}$, $267-278$ (1951)

Little, C. G., W. M. Rayton and R. B. Roof, Review of ionospheric effects at vhf and uhf, Proc. IRE 44, 992 1018 (1956).

Little, C. G., et al., Radio properties of the auroral ionosphere, Quarterly Progress Reports Nos. 1-5, 7, 8, Geophvsical Institute of the U. of Alaska (1958).

Little, C. G. and R. S. Lawrence, The use of polarization fading of satellite signals to study the electron content and irregularities in the ionosphere, J. Research NBS 64D, No. 4, 335-346 (1960).

Mills, B. Y. and A. B. Thomas, Observations of the source of radio-frequency radiation in the constellation of Cygnus, Aust. J. Sci. Res., Ser. A, 4, 158-171 (1951).

Munro, G. H., Travelling disturbances in the ionosphere, Proc. Roy. Soc. A, London, 202, p. 208 (1950).

Norton, K. A., et al, The probability distribution of the amplitude of a constant vector plus a Rayleigh-distributed vector, Proc. IRE 43, 1354-1361 (1955).

Ratcliffe, J. A., Some aspects of diffraction theory and their application to the ionosphere, Rep. Prog. Phys. 19, 188-267 (1956).

Reid, G. C., Variation with sidereal time of radio star scintillation rates, Can. J. Phys. 35, 1004-1016 (1957).

Rice, S. O., Mathematical analysis of random noise, Bell System Tech. J. 23, 24, 282-332, and 46-156 (1944 and 1945).

Ryle, M., A new radio interferometer and its application to the observation of weak radio stars, Proc. Roy. Soc. A, London, 211, 351-375 (1952).

Ryle, M. and A. Hewish, The effects of the terrestrial ionosphere on the radio waves from discrete sources in the galaxy, Mon. Not. Roy. Astron. Soc. 110, 381-394 (1950).

Smith, F. G., The determination of the position of a radio star, Mon. Not. Roy. Astron. Soc. 112, (1952).

Tukey, J., The sampling theory of power spectrum estimates, Symposium on Applications of Autocorrelation 
Analyses to Physical Problems, Woods Hole, Mass., Office of Naval Research, Washington, D.C. (1949).

Vitkevich, V. V. and Y. L. Kokurin, Irregular radio-wave refraction and extensive discontinuities in the ionosphere, Radiotekhnika i elektronika, 2, 826-832 (1957) (in Russian).

Vitkevich, V. V. and Y. L. Kokurin, Measurement of phase and amplitude fluctuations of radio waves which have traversed the ionosphere, Radiotekhnika i elektronika 3, 1373-1378 (1958) (in Russian).

Walker, H. M. and J. Lev, Statistical inference, Henry Holt and Co., 253-254 (1953).

Whitehead, J. D., The focussing of short radio waves reflected from the ionosphere, J. Atm. Terr. Phys. 9, 269275 (1956).
Wild, J. P. and J. A. Roberts, Regions of the ionosphere responsible for radio star scintillations, Nature 178, $377-378$, (1956).

Wild, J. P. and J. A. Roberts, The spectrum of radio-star scintillations and the nature of irregularities in the ionosphere, J. Atm. Terr. Phys. 8, 55-75 (1956).

Wright, R. W., J. R. Koster and N. J. Skinner, Spread F-layer echoes and radio-star scintillations, J. Atm. Terr. Phys. 8, 240-246 (1956).

Yeh, K. C. and G. W. Swenson, Jr., The scintillation of radio signals from satellites, J. Geophys. Res. 64, 2281-2286 (1959).

(Paper 65D4-137) 